Proc. Indian Acad. Sci. (Math. Sci.) Vol. 115, No. 3, August 2005, pp. 279-307.

(C) Printed in India

\title{
Degree-regular triangulations of torus and Klein bottle
}

\author{
BASUDEB DATTA and ASHISH KUMAR UPADHYAY \\ Department of Mathematics, Indian Institute of Science, Bangalore 560 012, India \\ E-mail: dattab@math.iisc.ernet.in; upadhyay@math.iisc.ernet.in
}

MS received 26 August 2004; revised 10 May 2005

\begin{abstract}
A triangulation of a connected closed surface is called weakly regular if the action of its automorphism group on its vertices is transitive. A triangulation of a connected closed surface is called degree-regular if each of its vertices have the same degree. Clearly, a weakly regular triangulation is degree-regular. In [8], Lutz has classified all the weakly regular triangulations on at most 15 vertices. In [5], Datta and Nilakantan have classified all the degree-regular triangulations of closed surfaces on at most 11 vertices.

In this article, we have proved that any degree-regular triangulation of the torus is weakly regular. We have shown that there exists an $n$-vertex degree-regular triangulation of the Klein bottle if and only if $n$ is a composite number $\geq 9$. We have constructed two distinct $n$-vertex weakly regular triangulations of the torus for each $n \geq 12$ and a $(4 m+2)$-vertex weakly regular triangulation of the Klein bottle for each $m \geq 2$. For $12 \leq n \leq 15$, we have classified all the $n$-vertex degree-regular triangulations of the torus and the Klein bottle. There are exactly 19 such triangulations, 12 of which are triangulations of the torus and remaining 7 are triangulations of the Klein bottle. Among the last 7 , only one is weakly regular.
\end{abstract}

Keywords. Triangulations of 2-manifolds; regular simplicial maps; combinatorially regular triangulations; degree-regular triangulations.

\section{Introduction and results}

Recall that a simplicial complex is a collection of non-empty finite sets (set of vertices) such that every non-empty subset of an element is also an element. For $i \geq 0$, the elements of size $i+1$ are called the $i$-simplices of the simplicial complex. 1 -simplices are also called the edges of the simplicial complex. For a simplicial complex $X$, the maximum of $k$ such that $X$ has a $k$-simplex is called the dimension of $X$. The set $V(X)$ of vertices of $X$ is called the vertex-set of $X$. A simplicial complex $X$ is called finite if $V(X)$ is finite.

If $X$ and $Y$ are two simplicial complexes, then a (simplicial) isomorphism from $X$ to $Y$ is a bijection $\varphi: V(X) \rightarrow V(Y)$ such that for $\sigma \subseteq V(X), \sigma$ is a simplex of $X$ if and only if $\varphi(\sigma)$ is a simplex of $Y$. Two simplicial complexes $X, Y$ are called (simplicially) isomorphic (and is denoted by $X \cong Y$ ) when such an isomorphism exists. We identify two complexes if they are isomorphic. An isomorphism from a simplicial complex $X$ to itself is called an automorphism of $X$. All the automorphisms of $X$ form a group, which is denoted by $\operatorname{Aut}(X)$.

A simplicial complex $X$ is usually thought of as a prescription for constructing a topological space (called the geometric carrier of $X$ and is denoted by $|X|$ ) by pasting together geometric simplices. Formally, $|X|$ is the subspace of $[0,1]^{V(X)}$ consisting of the functions 
$f: V(X) \rightarrow[0,1]$ such that the support $\{v \in V(X): f(v) \neq 0\}$ is a simplex of $X$ and $\sum_{v \in V(X)} f(v)=1$. If $\sigma$ is a simplex then $|\sigma|:=\left\{f \in|X|: \sum_{v \in \sigma} f(v)=1\right\}$ is called the geometric carrier of $\sigma$. We say that a simplicial complex $X$ triangulates a topological space $P$ (or $X$ is a triangulation of $P$ ) if $P$ is homeomorphic to $|X|$. A simplicial complex $X$ is called connected if $|X|$ is connected. A 2-dimensional simplicial complex is called a combinatorial 2-manifold if it triangulates a closed surface. A combinatorial 2-manifold $X$ is called orientable if $|X|$ is an orientable 2-manifold.

If $v$ is a vertex of a simplicial complex $X$, then the number of edges containing $v$ is called the degree of $v$ and is denoted by $\operatorname{deg}_{X}(v)$ (or $\operatorname{deg}(v)$ ). If the number of $i$-simplices of an $m$-dimensional finite simplicial complex $X$ is $f_{i}(X)(0 \leq i \leq m)$, then the number $\chi(X):=\sum_{i=0}^{m}(-1)^{i} f_{i}(X)$ is called the Euler characteristic of $X$. A simplicial complex is called neighbourly if each pair of vertices form an edge.

A combinatorially regular combinatorial 2-manifold is a connected combinatorial 2manifold with a flag-transitive automorphism group (a flag is a triple $(u, e, F)$, where $e$ is an edge of the face $F$ and $u$ is a vertex of $e$ ). A connected combinatorial 2-manifold $X$ is said to be weakly regular (or a weakly regular triangulation of $|X|$ ) if the automorphism group of $X$ acts transitively on $V(X)$. Clearly, a combinatorially regular combinatorial 2-manifold is weakly regular. Well-known examples of combinatorially regular combinatorial 2-manifolds are the boundaries of the tetrahedron, the octahedron, the icosahedron and the 6-vertex real projective plane $[4,5]$. The combinatorial manifolds $T_{3,3,0}$ and $T_{6,2,2}$ (in Examples 2 and 3) are combinatorially regular. Schulte and Wills [10,11] have constructed two combinatorially regular triangulations of the orientable surface of genus 3 . In [8], Lutz has shown that there are exactly 14 combinatorially regular combinatorial 2-manifolds on at most 22 vertices. By using computer, Lutz has shown the following:

\section{PROPOSITION 1}

There are exactly 77 weakly regular combinatorial 2 -manifolds on at most 15 vertices; 42 of these are orientable and 35 are non-orientable. Among these 77 combinatorial 2-manifolds, 20 are of Euler characteristic 0 . These 20 are $T_{7,1,2}, \ldots, T_{15,1,2}, T_{12,1,3}, \ldots, T_{15,1,3}$, $T_{12,1,4}, T_{15,1,4}, T_{15,1,5}, T_{6,2,2}, T_{3,3,0}, Q_{5,2}$ and $Q_{7,2}$ of Examples $1,2,3,6$.

A connected combinatorial 2-manifold $X$ is said to be degree-regular of type $d$ if each vertex of $X$ has degree $d$. A combinatorial 2-manifold $X$ is said to be degree-regular (or a degree-regular triangulation of $|X|$ ) if it is degree-regular of type $d$ for some $d$. So, trivial examples of degree-regular combinatorial 2-manifolds are weakly regular and neighbourly combinatorial 2-manifolds.

If $K$ is an $n$-vertex degree-regular of type $d$ combinatorial 2-manifold then $n d=2 f_{1}(K)$ $=3 f_{2}(K)$ and $\chi(K)=f_{0}(K)-f_{1}(K)+f_{2}(K)=n-\frac{n d}{2}+\frac{n d}{3}=\frac{n(6-d)}{6}$. So, if $\chi(K) \neq 0$ then only finitely many $(n, d)$ satisfies the above equation and hence only finitely many degree-regular combinatorial 2-manifolds of a given non-zero Euler characteristic. If $K$ is degree-regular and $\chi(K)>0$ then $(n, d)=(4,3),(6,4),(6,5)$ or $(12,5)$. For each $(n, d) \in\{(4,3),(6,4),(6,5),(12,5)\}$, there exists unique combinatorial 2-manifold, namely, the 4-vertex 2-sphere, the boundary of the octahedron, the 6-vertex real projective plane and the boundary of the icosahedron (see $[4,5])$. These 4 combinatorial 2-manifold are combinatorially regular. For the existence of degree-regular of type $d$ combinatorial 2-manifolds of negative Euler characteristic, $d$ must be at least 7. Since $\frac{n(6-d)}{6} \neq-1$ for $n>d \geq 7$, there does not exist any degree-regular combinatorial 2-manifolds of Euler characteristic -1 . If $\chi(K)=-2$ then $\left(f_{0}(K), d\right)=(12,7)$. In [6], we have seen that 
there are exactly 6 degree-regular triangulations of the orientable surface of genus 2 , three of which are weakly regular and none of them are combinatorially regular.

For the existence of an $n$-vertex neighbourly combinatorial 2-manifold, $n(n-1)$ must be divisible by 6 , equivalently, $n \equiv 0$ or $1 \bmod 3$. Ringel and Jungerman [7, 9] have shown that there exists neighbourly combinatorial 2 -manifolds on $3 k$ and $3 k+1$ vertices, for each $k \geq 2$. By using computer, Altshuler et al [3] have shown that there are exactly 59 orientable neighbourly combinatorial 2-manifolds on 12 vertices. In [2], Altshuler describe two operations by which one gets many neighbourly combinatorial 2-manifolds from one such combinatorial 2-manifold on the same number of vertices. Using this he has constructed 40615 distinct non-orientable neighbourly combinatorial 2-manifolds on 12 vertices.

Here we are interested in the cases when the Euler characteristic is 0 (i.e., triangulations of the torus and the Klein bottle). Clearly, if $K$ is an $n$-vertex degree-regular combinatorial 2-manifold and $\chi(K)=0$ then $n>d=6$. From [5], we know the following:

\section{PROPOSITION 2}

(a) For each $n \geq 7$, there exists an $n$-vertex weakly regular triangulation of the torus.

(b) For each $k, l \geq 3$, there exists a kl-vertex degree-regular triangulation of the Klein bottle.

\section{PROPOSITION 3}

There are exactly 27 degree-regular combinatorial 2-manifolds on at most 11 vertices; 8 of which are of Euler characteristic 0 . These 8 are $T_{7,1,2}, \ldots, T_{11,1,2}, T_{3,3,0}, B_{3,3}$ and $Q_{5,2}$ of Examples 1,3,4,6.

Here we prove the following.

Theorem 1. Any degree-regular triangulation of the torus is weakly regular.

Theorem 2. There exists an n-vertex degree-regular triangulation of the Klein bottle if and only if $n$ is a composite number $\geq 9$.

\section{Theorem 3.}

(a) For each $n \geq 12$ there exists atleast two distinct $n$-vertex weakly regular triangulations of the torus.

(b) For each $n \geq 18$ there exists atleast three distinct $n$-vertex weakly regular triangulations of the torus.

(c) For each $m \geq 2$ there exists a $(4 m+2)$-vertex weakly regular triangulation of the Klein bottle.

Theorem 4. Let $T_{n, 1, k}$ be as in Example 1. For a prime $n \geq 7$, if $M$ is an $n$-vertex weakly regular triangulation of the torus then $M$ is isomorphic to $T_{n, 1, k}$ for some $k$.

\section{COROLLARY 5}

(a) For $n=13$ or 17, there are exactly 2 distinct $n$-vertex degree-regular combinatorial 2-manifolds of Euler characteristic 0 . These are $T_{n, 1,2}$ and $T_{n, 1,3}$. 
(b) There are exactly 3 distinct 19-vertex degree-regular combinatorial 2-manifolds of Euler characteristic 0 . These are $T_{19,1,2}, T_{19,1,3}$ and $T_{19,1,7}$.

From Theorem 1 and Proposition 1 we know all the degree-regular triangulations of the torus on at most 15 vertices. Here we present (without using computer) the following:

Theorem 6. Let $M$ be an n-vertex degree-regular combinatorial 2-manifold of Euler characteristic 0 . If $n=12,14$ or 15 then $M$ is isomorphic to $T_{12,1,2}, \ldots, T_{12,1,4}, T_{6,2,2}$, $T_{14,1,2}, T_{14,1,3}, T_{15,1,2}, \ldots, T_{15,1,5}, Q_{7,2}, Q_{5,3}, B_{3,4}, B_{4,3}, B_{3,5}, B_{5,3}$ or $K_{3,4}$. These 17 combinatorial 2-manifolds are pairwise non-isomorphic. The first 10 triangulate the torus and the remaining 7 triangulate the Klein bottle. Among the last 7 , only $Q_{7,2}$ is weakly regular.

\section{Examples}

In this section we present some degree-regular combinatorial 2-manifolds of Euler characteristic 0 . First we give some definitions and notations which will be used throughout the paper.

A 2-simplex in a 2-dimensional simplicial complex is also said to be a face. We denote a face $\{u, v, w\}$ by $u v w$. We also denote an edge $\{u, v\}$ by $u v$.

A graph is a simplicial complex of dimension at most one. The complete graph on $n$ vertices is denoted by $K_{n}$. Disjoint union of $m$ copies of $K_{n}$ is denoted by $m K_{n}$. A graph without any edge is called a null graph. An $n$-vertex null graph is denoted by $\emptyset_{n}$.

If $G$ is a graph and $n \geq 0$ is an integer then we define the graph $G_{n}(G)$ as follows. The vertices of $G_{n}(G)$ are the vertices of $G$. Two vertices $u$ and $v$ form an edge in $G_{n}(G)$ if the number of common neighbours of $u$ and $v$ is $n$. Clearly, if $G$ and $H$ are isomorphic then $G_{n}(G)$ and $G_{n}(H)$ are isomorphic for all $n \geq 0$.

A connected finite graph is called a cycle if the degree of each vertex is 2 . An $n$-cycle is a cycle on $n$ vertices and is denoted by $C_{n}$ (or by $C_{n}\left(a_{1}, \ldots, a_{n}\right)$ if the edges are $\left.a_{1} a_{2}, \ldots, a_{n-1} a_{n}, a_{n} a_{1}\right)$. Disjoint union of $m$ copies of $C_{n}$ is denoted by $m C_{n}$.

For a simplicial complex $K$, the graph consisting of the edges and vertices of $K$ is called the edge-graph of $K$ and is denoted by $\operatorname{EG}(K)$. The complement of $\operatorname{EG}(K)$ is called the non-edge graph of $K$ and is denoted by $\operatorname{NEG}(K)$. Let $K$ be a simplicial complex with vertex-set $V(K)$. If $U \subseteq V(K)$ then the induced subcomplex of $K$ on $U$, denoted by $K[U]$, is the subcomplex whose simplices are those of $K$ which are subsets of $U$.

If $v$ is a vertex of a simplicial complex $X$, then the link of $v$ in $X$, denoted by $\operatorname{lk}_{X}(v)$ (or $\operatorname{lk}(v)$ ), is the simplicial complex $\{\tau \in X: v \notin \tau,\{v\} \cup \tau \in X\}$. If $v$ is a vertex of a simplicial complex $X$, then the star of $v$ in $X$, denoted by $\operatorname{st}_{X}(v)$ (or st $(v)$ ), is the simplicial complex $\left\{\{v\}, \tau, \tau \cup\{v\}: \tau \in \mathrm{lk}_{X}(v)\right\}$. Clearly, a finite simplicial complex $K$ is a combinatorial 2-manifold if and only if $\operatorname{lk}_{K}(v)$ is a cycle for each vertex $v$ of $K$.

Example 1. A series of weakly regular orientable combinatorial 2-manifolds of Euler characteristic 0 . For each $n \geq 7$ and each $k \in\left\{2, \ldots,\left\lfloor\frac{n-3}{2}\right\rfloor\right\} \cup\left\{\left\lceil\frac{n+1}{2}\right\rceil, \ldots, n-3\right\}$,

$$
T_{n, 1, k}=\{\{i, i+k, i+k+1\},\{i, i+1, i+k+1\}: 1 \leq i \leq n\},
$$


where $V\left(T_{n, 1, k}\right)=\{1, \ldots, n\}$. Since $\operatorname{lk}(i)=C_{6}(i+k, n+i-1, n+i-k-1, n+i-$ $k, i+1, i+k+1), T_{n, 1, k}$ is a combinatorial 2-manifold. Clearly, $T_{n, 1, k}$ triangulates the torus and hence it is orientable. Since $\mathbb{Z}_{n}$ acts transitively (by addition) on vertices, $T_{n, 1, k}$ is weakly regular. (Here addition is modulo $n$.) In [1], Altshuler has shown that $T_{n, 1, k}$ is a subcomplex of an $n$-vertex cyclic polytopal 3 -sphere.

Lemma 2.1. Let $T_{n, 1, k}$ be as above. We have the following:

(a) $T_{n, 1, k}=T_{n, 1, n-k-1}$ for all $n$ and $k$.

(b) $T_{n, 1,2} \neq T_{n, 1,3}$ for all $n \geq 12$.

(c) $T_{n, 1,2} ¥ T_{n, 1,4} ¥ T_{n, 1,3}$ for all $n \geq 20$.

(d) $T_{12,1,2} ¥ T_{12,1,4} ¥ T_{12,1,3}$.

(e) $T_{13,1,4} \cong T_{13,1,2} \cong T_{13,1,5}$.

(f) $T_{15,1, k} \neq T_{15,1, j}$ for $j, k \in\{2,3,4,5\}$ and $j \neq k$.

(g) $T_{16,1,5} \cong T_{16,1,2} ¥ T_{16,1,6} ¥ T_{16,1,3} \cong T_{16,1,4}$.

(h) $T_{17,1,5} \cong T_{17,1,7} \cong T_{17,1,2}$ and $T_{17,1,4} \cong T_{17,1,6} \cong T_{17,1,3}$.

(i) $T_{18,1,6} \cong T_{18,1,5}$ and $T_{18,1, k} ¥ T_{18,1, j}$ for $j, k \in\{2,3,4,5,7\}, j \neq k$.

(j) $T_{19,1,6} \cong T_{19,1,8} \cong T_{19,1,2} ¥ T_{19,1,7} \neq T_{19,1,3} \cong T_{19,1,4} \cong T_{19,1,5}$.

(k) $T_{20,1,6} \cong T_{20,1,2}, T_{20,1,7} \cong T_{20,1,3}$ and $T_{20,1, k} ¥ T_{20,1, j}$ for $j, k \in\{2,3,4,5,8\}, j \neq k$.

Proof. Observe that $\mathrm{lk}_{T_{n, 1, k}}(i)=C_{6}(i+1, i+k+1, i+k, n+i-1, n+i-k-$ $1, n+i-k)=\operatorname{lk}_{T_{n, 1, n-k-1}}(i)$. So, the faces in both $T_{n, 1, k}$ and $T_{n, 1, n-k-1}$ are same. This proves (a).

Then $G_{4}\left(\operatorname{EG}\left(T_{n, 1,2}\right)\right)=C_{n}(1, \ldots, n)$ for $n \geq 11$ and $G_{4}\left(\operatorname{EG}\left(T_{n, 1,3}\right)\right)$ is a null graph for $n=13$ and for $n \geq 15$. Also, $G_{4}\left(\operatorname{EG}\left(T_{14,1,2}\right)\right)$ is $7 K_{2}$. So, $T_{n, 1,2} \neq T_{n, 1,3}$ for all $n \geq 13$.

Observe that $G_{4}\left(\operatorname{EG}\left(T_{12,1,3}\right)\right)$ is a 12-cycle with edges $\{i, i+5\}, 1 \leq i \leq 12$. So, the edges of $G_{4}\left(\operatorname{EG}\left(T_{12,1,3}\right)\right)$ are non-edges of $T_{12,1,2}$. But $G_{4}\left(\operatorname{EG}\left(T_{12,1,2}\right)\right)$ is a subgraph of $\mathrm{EG}\left(T_{12,1,2}\right)$. So, $T_{12,1,2} ¥ T_{12,1,3}$. This proves (b).

For all $n \geq 20, G_{3}\left(\operatorname{EG}\left(T_{n, 1,4}\right)\right)$ is a null graph, but $\{i, i+2\}$ is an edge in $G_{3}\left(\operatorname{EG}\left(T_{n, 1,3}\right)\right)$. So, $G_{3}\left(\operatorname{EG}\left(T_{n, 1,4}\right)\right) \varsubsetneqq G_{3}\left(\operatorname{EG}\left(T_{n, 1,3}\right)\right)$ and hence $T_{n, 1,4} ¥ T_{n, 1,3}$ for $n \geq 20$.

Again, for all $n \geq 20, G_{4}\left(\operatorname{EG}\left(T_{n, 1,4}\right)\right)=\emptyset_{n}$. So, $G_{4}\left(\operatorname{EG}\left(T_{n, 1,4}\right)\right) \not G_{4}\left(\operatorname{EG}\left(T_{n, 1,2}\right)\right)$ and hence $T_{n, 1,4} ¥ T_{n, 1,2}$ for $n \geq 20$. This proves (c).

Observe that $G_{4}\left(\operatorname{EG}\left(T_{12,1,4}\right)\right)=3 K_{4}$ (with edges $\left.\{i, j\}, i-j \equiv 0(\bmod 3)\right)$. Since, $G_{4}\left(\operatorname{EG}\left(T_{12,1,2}\right)\right)$ and $G_{4}\left(\operatorname{EG}\left(T_{12,1,3}\right)\right)$ are 12 -cycles, $T_{12,1,2} \neq T_{12,1,4} ¥ T_{12,1,3}$. This proves (d).

The map $i \mapsto 4 i(\bmod 13)$ defines an isomorphism from $T_{13,1,2}$ to $T_{13,1,4}$ and the map $i \mapsto 7 i(\bmod 13)$ defines an isomorphism from $T_{13,1,2}$ to $T_{13,1,5}$. This proves (e).

Observe that $G_{0}\left(\operatorname{EG}\left(T_{15,1,2}\right)\right)$ is a 15 -cycle (with edges $\{i, i+7\}, 1 \leq i \leq 15$ ) and $G_{0}\left(\operatorname{EG}\left(T_{15,1, i}\right)\right)$ is a null graph for $i=3,4,5$. So, $T_{15,1, i} \neq T_{15,1,2}$ for $i=3$, 4 or 5 . Again, $G_{4}\left(\operatorname{EG}\left(T_{15,1,3}\right)\right)$ is a null graph, $G_{4}\left(\operatorname{EG}\left(T_{15,1,4}\right)\right)=3 C_{5}$ (with edges $\{i, i+6\}$, $1 \leq i \leq 15)$ and $G_{4}\left(\operatorname{EG}\left(T_{15,1,5}\right)\right)=C_{15}$ (with edges $\{i, i+4\}, 1 \leq i \leq 15$ ). So, $T_{15,1,3} ¥ T_{15,1,4} ¥ T_{15,1,5} ¥ T_{15,1,3}$. These prove (f).

The map $i \mapsto 3 i(\bmod 16)$ defines an isomorphism from $T_{16,1,4}$ to $T_{16,1,3}$ and an isomorphism from $T_{16,1,5}$ to $T_{16,1,2}$.

Observe that $G_{4}\left(\operatorname{EG}\left(T_{16,1,6}\right)\right)=8 K_{2}$ (with edges $\{i, i+8\}, 1 \leq i \leq 8$ ). Since $G_{4}\left(\operatorname{EG}\left(T_{16,1,2}\right)\right)=C_{16}$ and $G_{4}\left(\operatorname{EG}\left(T_{16,1,3}\right)\right)$ is a null graph, $T_{16,1,2} ¥ T_{16,1,6} ¥ T_{16,1,3}$. This proves $(\mathrm{g})$. 
The map $i \mapsto 14 i(\bmod 17)$ defines an isomorphism from $T_{17,1,5}$ to $T_{17,1,2}$. The map $i \mapsto 2 i(\bmod 17)$ defines an isomorphism from $T_{17,1,7}$ to $T_{17,1,2}$. The map $i \mapsto 13 i(\bmod$ 17) defines an isomorphism from $T_{17,1,4}$ to $T_{17,1,3}$. The map $i \mapsto 3 i(\bmod 17)$ defines an isomorphism from $T_{17,1,6}$ to $T_{17,1,3}$. This proves (h).

The map $i \mapsto 5 i(\bmod 18)$ defines an isomorphism from $T_{18,1,6}$ to $T_{18,1,5}$.

Now, $G_{3}\left(\operatorname{EG}\left(T_{18,1,3}\right)\right)=2 C_{9}($ with edges $\{i, i+2\}, 1 \leq i \leq 18)$ and $G_{3}\left(\operatorname{EG}\left(T_{18,1,7}\right)\right)=$ $9 K_{2}$ (with edges $\left.\{i, i+9\}, 1 \leq i \leq 9\right)$. So, $T_{18,1,3} \neq T_{18,1,7}$. Again, $G_{4}\left(\operatorname{EG}\left(T_{18,1,2}\right)\right)=$ $C_{18}(1,2, \ldots, 18) \subseteq \mathrm{EG}\left(T_{18,1,2}\right), G_{4}\left(\mathrm{EG}\left(T_{18,1,3}\right)\right)$ and $G_{4}\left(\mathrm{EG}\left(T_{18,1,7}\right)\right)$ are null graphs, $G_{4}\left(\operatorname{EG}\left(T_{18,1,4}\right)\right)=9 K_{2}$ (with edges $\left.\{i, i+9\}, 1 \leq i \leq 9\right)$ and $G_{4}\left(\operatorname{EG}\left(T_{18,1,5}\right)\right)=C_{18}$ (with edges $\{i, i+7\}, 1 \leq i \leq 18)$. Thus, $G_{4}\left(\operatorname{EG}\left(T_{18,1,5}\right)\right)$ is not a subgraph of $\operatorname{EG}\left(T_{18,1,5}\right)$. These imply (i).

The map $i \mapsto 3 i$ (mod 19) defines an isomorphism from $T_{19,1,5}$ to $T_{19,1,3}$. The map $i \mapsto 15 i(\bmod 19)$ defines an isomorphism from $T_{19,1,4}$ to $T_{19,1,3}$. The map $i \mapsto 6 i(\bmod$ 19) defines an isomorphism from $T_{19,1,2}$ to $T_{19,1,6}$. The map $i \mapsto 9 i(\bmod 19)$ defines an isomorphism from $T_{19,1,2}$ to $T_{19,1,8}$.

The graph $G_{4}\left(\operatorname{EG}\left(T_{19,1,7}\right)\right)$ is null, whereas $G_{4}\left(\operatorname{EG}\left(T_{19,1,2}\right)\right)=C_{19}$. So, $T_{19,1,7} \not$ $T_{19,1,2}$. Again, $G_{0}\left(\operatorname{EG}\left(T_{19,1,7}\right)\right)$ is null, whereas $G_{0}\left(\operatorname{EG}\left(T_{19,1,3}\right)\right)=C_{19}$ (with edges $\{i, i+9\}, 1 \leq i \leq 19)$. So, $T_{19,1,7} ¥ T_{19,1,3}$. This proves $(\mathrm{j})$.

The map $i \mapsto 3 i(\bmod 20)$ defines an isomorphism from $T_{20,1,6}$ to $T_{20,1,2}$ and an isomorphism from $T_{20,1,7}$ to $T_{20,1,3}$.

Observe that $G_{4}\left(\operatorname{EG}\left(T_{20,1,2}\right)\right)=C_{20}, G_{4}\left(\operatorname{EG}\left(T_{20,1,3}\right)\right), G_{4}\left(\operatorname{EG}\left(T_{20,1,4}\right)\right), G_{4}$ $\left(\operatorname{EG}\left(T_{20,1,5}\right)\right)$ are null graphs and $G_{4}\left(\operatorname{EG}\left(T_{20,1,8}\right)\right)=10 K_{2}$ (with edges $\{i, i+10\}$, $1 \leq i \leq 10)$. So, $T_{20,1,2} ¥ T_{20,1, i}$ for $i=3,4,5,8$ and $T_{20,1,8} ¥ T_{20,1, i}$ for $i=3,4,5$.

Again, $G_{3}\left(\operatorname{EG}\left(T_{20,1,3}\right)\right)=2 C_{10}$ (with edges $\left.\{i, i+2\}, 1 \leq i \leq 20\right)$ but $G_{3}\left(\operatorname{EG}\left(T_{20,1,4}\right)\right.$ ) and $G_{3}\left(\operatorname{EG}\left(T_{20,1,5}\right)\right)$ are null graphs. So, $T_{20,1,3} ¥ T_{20,1, i}$ for $i=4,5$.

Finally, if possible, let $\varphi$ be an isomorphism from $T_{20,1,4}$ to $T_{20,1,5}$. Then $\varphi$ induces isomorphism between $G_{n}\left(\operatorname{EG}\left(T_{20,1,4}\right)\right)$ and $G_{n}\left(\operatorname{EG}\left(T_{20,1,5}\right)\right)$ for each $n$. Since, Aut $\left(T_{20,1,4}\right)$ acts transitively on $V\left(T_{20,1,4}\right)$, we can assume that $\varphi(20)=20$. Since $G_{0}\left(\operatorname{EG}\left(T_{20,1,4}\right)\right)=$ $C_{20}(20,7,14, \ldots, 13)$ and $G_{0}\left(\operatorname{EG}\left(T_{20,1,5}\right)\right)=C_{20}(20,3,6, \ldots, 17), \varphi(7)=17$ or 3 . If $\varphi(7)=17$ then $\varphi(14)=14, \varphi(1)=11, \ldots$ In that case, $\varphi(\{20,1\})=\{20,11\}$. This is a contradiction since $\{20,1\}$ is an edge in $T_{20,1,4}$ but $\{20,11\}$ is not an edge in $T_{20,1,5}$. Similarly, we get a contradiction if $\varphi(7)=3$. This proves $(\mathrm{k})$.

Let $D_{n}$ denote the dihedral group of order $2 n$ and $\mathbb{Z}_{m^{2}-m+1}: \mathbb{Z}_{6}:=\left\langle\rho, \mu: \rho^{m^{2}-m+1}=\right.$ $\left.1=\mu^{6}, \mu^{-1} \rho \mu=\rho^{m}\right\rangle$ for $m \geq 3$. In [8], Lutz has shown that $\operatorname{Aut}\left(T_{n, 1, k}\right)=D_{n}$ for $(n, k)=(9,2), \ldots,(15,2),(12,3),(14,3),(15,5), \operatorname{Aut}\left(T_{12,1,4}\right)=D_{4} \times D_{3}$, $\operatorname{Aut}\left(T_{15,1, k}\right)=D_{5} \times D_{3}$ for $k=3,4$ and $\operatorname{Aut}\left(T_{m^{2}-m+1,1, m-1}\right)=\mathbb{Z}_{m^{2}-m+1}: \mathbb{Z}_{6}$ for $m=3,4$. Here we prove the following.

\section{Lemma 2.2.}

(a) $D_{n}$ acts face-transitively on $T_{n, 1, k}$ for all $n \geq 7$ and for all $k$.

(b) $\operatorname{Aut}\left(T_{n, 1,2}\right)=D_{n}$ for all $n \geq 9$.

(c) $D_{2 m} \times D_{m+1} \leq \operatorname{Aut}\left(T_{2 m^{2}+2 m, 1,2 m}\right)$ for $m \geq 2$.

(d) $D_{m+1} \times D_{m-1} \leq \operatorname{Aut}\left(T_{m^{2}-1,1, m-1}\right)$ for $m \geq 4$.

(e) $D_{m+1} \times D_{m-1} \leq \operatorname{Aut}\left(T_{m^{2}-1,1, m}\right)$ for $m \geq 4$.

(f) $\mathbb{Z}_{m^{2}-m+1}: \mathbb{Z}_{6} \leq \operatorname{Aut}\left(T_{m^{2}-m+1,1, m-1}\right)$ for $m \geq 3$.

Here $H \leq G$ means $G$ has a subgroup isomorphic to $H$. 
Proof. Let $\alpha_{n}, \beta_{n}: V\left(T_{n, 1, k}\right) \rightarrow V\left(T_{n, 1, k}\right)$ be given by $\alpha_{n}(i)=i+1$ and $\beta_{n}(i)=n-i$ (modulo $n$ ). Let $A_{n, k, i}:=\{i, i+1, i+k+1\}$ and $B_{n, k, i}:=\{i, i+k, i+k+1\}$. Then $\alpha_{n}\left(A_{n, k, i}\right)=A_{n, k, i+1}, \alpha_{n}\left(B_{n, k, i}\right)=B_{n, k, i+1}, \beta_{n}\left(A_{n, k, i}\right)=B_{n, k, n-i-k-1}$ and $\beta_{n}\left(B_{n, k, i}\right)=A_{n, k, n-i-k-1}$. So, $\alpha_{n}, \beta_{n} \in \operatorname{Aut}\left(T_{n, 1, k}\right)$. Clearly, the order of $\alpha_{n}$ is $n$, the order of $\beta_{n}$ is 2 and $\beta_{n} \alpha_{n} \beta_{n}=\alpha_{n}^{-1}$. Thus, $\left\langle\alpha_{n}, \beta_{n}\right\rangle$ is isomorphic to $D_{n}$. Clearly, the action of $\left\langle\alpha_{n}, \beta_{n}\right\rangle$ on $T_{n, 1, k}$ is transitive on the faces. This proves (a).

For $n \geq 11, G_{4}\left(\operatorname{EG}\left(T_{n, 1,2}\right)\right)=C_{n}(1,2, \ldots, n)$. Therefore, $\left\langle\alpha_{n}, \beta_{n}\right\rangle \leq \operatorname{Aut}\left(T_{n, 1,2}\right) \leq$ $\operatorname{Aut}\left(G_{4}\left(\mathrm{EG}\left(T_{n, 1,2}\right)\right)\right)=\operatorname{Aut}\left(C_{n}(1, \ldots, n)\right)=\left\langle\alpha_{n}, \beta_{n}\right\rangle$. Thus, Aut $\left(T_{n, 1,2}\right)=\left\langle\alpha_{n}, \beta_{n}\right\rangle$ for $n \geq 11$. Since $G_{2}\left(\operatorname{EG}\left(T_{10,1,2}\right)\right)=C_{10}(1,4,7,10,3,6,9,2,5,8),\left\langle\alpha_{10}^{3}, \beta_{10}\right\rangle \leq$ $\operatorname{Aut}\left(T_{10,1,2}\right) \leq \operatorname{Aut}\left(G_{2}\left(\operatorname{EG}\left(T_{10,1,2}\right)\right)\right)=\operatorname{Aut}\left(C_{10}(1,4, \ldots, 5,8)\right)=\left\langle\alpha_{10}^{3}, \beta_{10}\right\rangle$. Thus, $\operatorname{Aut}\left(T_{10,1,2}\right)=\left\langle\alpha_{10}^{3}, \beta_{10}\right\rangle=\left\langle\alpha_{10}, \beta_{10}\right\rangle$. Observe that $\operatorname{NEG}\left(T_{9,1,2}\right)=C_{9}(1,5,9,4,8,3$, 7, 2, 6). Therefore, $\left\langle\alpha_{9}^{4}, \beta_{9}\right\rangle \leq \operatorname{Aut}\left(T_{9,1,2}\right) \leq \operatorname{Aut}\left(\operatorname{NEG}\left(T_{9,1,2}\right)\right)=\operatorname{Aut}\left(C_{9}(1,5,9,4,8,3\right.$, $7,2,6))=\left\langle\alpha_{9}^{4}, \beta_{9}\right\rangle$. Thus, Aut $\left(T_{9,1,2}\right)=\left\langle\alpha_{9}^{4}, \beta_{9}\right\rangle=\left\langle\alpha_{9}, \beta_{9}\right\rangle$. This proves (b).

Let $\alpha, \beta, \gamma, \delta: V\left(T_{2 m^{2}+2 m, 1,2 m}\right) \rightarrow V\left(T_{2 m^{2}+2 m, 1,2 m}\right)$ be given by $\alpha(i)=i+m+1$, $\beta(i)=i+2 m, \gamma(i)=(2 m+1) i$ and $\delta(i)=\left(2 m^{2}-1\right) i\left(\right.$ i.e., $\left.\alpha=\alpha_{2 m^{2}+2 m}^{m+1}, \beta=\alpha_{2 m^{2}+2 m}^{2 m}\right)$. Then $\alpha, \beta, \gamma, \delta \in \operatorname{Aut}\left(T_{2 m^{2}+2 m, 1,2 m}\right)$, order of $\alpha$ is $2 m$, order of $\beta$ is $m+1$, order of $\gamma$ is 2 , order of $\delta$ is $2, \alpha \beta=\beta \alpha, \alpha \gamma=\gamma \alpha, \beta \delta=\delta \beta, \gamma \delta=\delta \gamma=\beta_{2 m^{2}+2 m}, \delta \alpha \delta=\alpha^{-1}$, $\gamma \beta \gamma=\beta^{-1}$. Therefore, $\langle\alpha, \beta, \gamma, \delta\rangle=\langle\alpha, \delta\rangle \times\langle\beta, \gamma\rangle \cong D_{2 m} \times D_{m+1}$. This proves (c).

Claim. If $m^{2} \equiv 1(\bmod n)$, then $\mu(i)=m i$ and $v(i)=(n-m) i$ define two distinct involutions (automorphisms of order 2) of $T_{n, 1, k}$ for each $k \in\{m-1, m\}$.

Let $A_{n, k, i}$ and $B_{n, k, i}$ be as above. Then $\mu\left(A_{n, m-1, i}\right)=A_{n, m-1, m i}, \mu\left(B_{n, m-1, i}\right)=$ $B_{n, m-1, m i-m+1}, \mu\left(A_{n, m, i}\right)=B_{n, m, m i}$ and $\mu\left(B_{n, m, i}\right)=A_{n, m, m i}$. Thus $\mu \in \operatorname{Aut}\left(T_{n, 1, k}\right)$ and hence $v=\beta_{n} \mu=\mu \beta_{n} \in \operatorname{Aut}\left(T_{n, 1, k}\right)$ for $k=m-1, m$. Since $\mu^{-1}=\mu, v^{-1}=v$ and $\mu \nu=\beta_{n} \neq$ the identity, $\mu \neq v$. This proves the claim.

For $k=m-1, m$, let $\alpha, \lambda, \mu, v: V\left(T_{m^{2}-1,1, k}\right) \rightarrow V\left(T_{m^{2}-1,1, k}\right)$ be given by $\alpha(i)=$ $i+m+1, \lambda(i)=i+m-1, \mu(i)=m i$ and $v(i)=(n-m) i$ (i.e., $\alpha=\alpha_{m^{2}-1}^{m+1}, \lambda=\alpha_{m^{2}-1}^{m-1}$ ). Then $\alpha$ and $\lambda$ are automorphisms of $T_{m^{2}-1,1, k}$. Also, by the above claim, $\mu$ and $v$ are distinct automorphisms of $T_{m^{2}-1,1, k}$. Clearly, the orders of $\alpha, \lambda, \mu$ and $v$ are $m-1, m+1$, 2 and 2 respectively. Again, $\alpha \mu=\mu \alpha, \alpha \lambda=\lambda \alpha, \lambda \nu=v \lambda, \mu \nu=v \mu, v \alpha v=\alpha^{-1}$ and $\mu \lambda \mu=\lambda^{-1}$. Thus, $\langle\alpha, \lambda, \mu, v\rangle=\langle\lambda, \mu\rangle \times\langle\alpha, v\rangle \cong D_{m+1} \times D_{m-1}$. This proves (d) and (e).

Let $\sigma: V\left(T_{m^{2}-m+1,1, m-1}\right) \rightarrow V\left(T_{m^{2}-m+1,1, m-1}\right)$ be given by $\sigma(i)=m i$. Then $\sigma\left(A_{m^{2}-m+1, m-1, i}\right)=B_{m^{2}-m+1, m-1, m i}$ and $\sigma\left(B_{m^{2}-m+1, m-1, i}\right)=A_{m^{2}-m+1, m-1, m i-1}$. Thus, $\sigma \in \operatorname{Aut}\left(T_{m^{2}-m+1,1, m-1}\right)$. Since 6 is the smallest positive integer $n$ for which $m^{n}-1$ is divisible by $m^{2}-m+1$, the order of $\sigma$ is 6. Now, if $\rho=\alpha_{m^{2}-m+1}$ (i.e., $\rho(i)=i+1$ ) then $\rho$ is an automorphism of order $m^{2}-m+1$ and $\left(\sigma \circ \rho \circ \sigma^{-1}\right)(i)=m((1-m) i+1)=$ $i+m=\rho^{m}(i)$. Thus, $\langle\rho, \sigma\rangle=\mathbb{Z}_{m^{2}-m+1}: \mathbb{Z}_{6}$. This proves (f).

Example 2. A series of weakly regular orientable combinatorial 2-manifolds of Euler characteristic 0 . For each $n \geq 4$ and each $k=1, \ldots, n-3$,

$$
T_{n, 2, k}=\left\{u_{i} u_{i+1} v_{i+1}, u_{i} v_{i} v_{i+1}, u_{i+k} u_{i+k+1} v_{i}, u_{i+k+1} v_{i} v_{i+1}: 1 \leq i \leq n\right\},
$$

where $V\left(T_{n, 2, k}\right)=\left\{u_{1}, \ldots, u_{n}\right\} \cup\left\{v_{1}, \ldots, v_{n}\right\}$. (Addition in the subscripts are modulo n.) Since $\operatorname{lk}\left(u_{i}\right)=C_{6}\left(u_{i-1}, v_{i}, v_{i+1}, u_{i+1}, v_{n+i-k-1}, v_{n+i-k}\right)$ and $\operatorname{lk}\left(v_{j}\right)=$ $C_{6}\left(v_{j-1}, u_{j-1}, u_{j}, v_{j+1}, u_{j+k+1}, u_{j+k}\right), T_{n, 2, k}$ is a degree-regular combinatorial 2manifold on $2 n$ vertices. Clearly, $T_{n, 2, k}$ triangulates the torus and hence it is orientable. 
If $\alpha, \beta: V\left(T_{n, 2, k}\right) \rightarrow V\left(T_{n, 2, k}\right)$ are the maps given by $\alpha\left(u_{i}\right)=u_{i+1}, \alpha\left(v_{i}\right)=v_{i+1}$, $\beta\left(u_{i}\right)=v_{i}, \beta\left(v_{i}\right)=u_{i+k}$ for $1 \leq i \leq n$, then $\alpha$ and $\beta$ are automorphisms of $T_{n, 2, k}$ and hence $\langle\alpha, \beta\rangle$ is a subgroup of $\operatorname{Aut}\left(T_{n, 2, k}\right)$. Clearly, $\langle\alpha, \beta\rangle$ acts transitively on vertices. Thus $T_{n, 2, k}$ is weakly regular.

Lemma 2.3. Let $T_{n, 1, j}$ and $T_{m, 2, k}$ be as in Examples 1,2. We have the following:

(a) If $n$ and $k$ are relatively prime or $n$ and $k+2$ are relatively prime then $T_{n, 2, k}$ is isomorphic to $T_{2 n, 1, j}$ for some $j$.

(b) $T_{6,2,2} ¥ T_{12,1, i}$ for all $i$.

(c) $T_{8,2,4} \cong T_{8,2,2} ¥ T_{16,1, i}$ for all $i$.

Proof. If $(n, k)=1$ and $k \leq n-3$, then there exists $p \in\{1, \ldots, n-2\}$ such that $p k \equiv 1(\bmod n)$. Since $k \leq n-3$ and $n \geq 4,2 p \neq n, n-1$. $(2 p=n$ implies $2 \equiv 2 p k \equiv 0(\bmod n)$, a contradiction. $2 p=n-1$ implies $2 \equiv 2 p k \equiv n-k$. This implies $k \equiv n-2(\bmod n)$ and hence $k=n-2$.) Let $\varphi: V\left(T_{n, 2, k}\right) \rightarrow\{1, \ldots, 2 n\}$ be given by $\varphi\left(u_{i}\right)=1+2 p(i-1)$ and $\varphi\left(v_{i}\right)=2+2 p(i-1)$ (modulo $\left.2 n\right)$. Since $(n, p)=1, \varphi$ is a bijection. Now, $\varphi\left(u_{i} u_{i+1} v_{i+1}\right)=\{1+2(i-1) p, 1+2 i p, 2+2 i p\}$, $\varphi\left(u_{i} v_{i} v_{i+1}\right)=\{1+2(i-1) p, 2+2(i-1) p, 2+2 i p\}, \varphi\left(u_{i+k} u_{i+k+1} v_{i}\right)=\{1+2(i-1) p+$ $2,1+2 i p+2,2+2(i-1) p\}=\{2(i-1) p+2,2(i-1) p+3,2 i p+3\}, \varphi\left(u_{i+k+1} v_{i} v_{i+1}\right)=$ $\{2 i p+3,2(i-1) p+2,2+2 i p\}=\{2(i-1) p+2,2 i p+2,2 i p+3\} \in T_{2 n, 1,2 p}$. This shows that $\varphi: T_{n, 2, k} \rightarrow T_{2 n, 1,2 p}$ is an isomorphism.

If $(n, k+2)=1$ then assume that $(n, k) \neq 1$ (otherwise there is nothing to prove). Let $p(k+2) \equiv 1(\bmod n)$. Observe that $2 p \neq n, n-1 .(2 p=n$ implies $2 \equiv 2 p(k+2) \equiv 0$ $(\bmod n)$, a contradiction. $2 p=n-1$ implies $2 \equiv 2 p(k+2) \equiv(n-1)(k+2) \equiv n-k-2$. This implies $k \equiv n-4(\bmod n)$ and hence $k=n-4$. Since $(n, k) \neq 1, n$ and $k$ are even and hence $(n, k+2) \neq 1$, a contradiction.) Let $\psi: V\left(T_{n, 2, k}\right) \rightarrow\{1, \ldots, 2 n\}$ be given by $\psi\left(u_{i}\right)=1+2 p(i-1)$ and $\psi\left(v_{i}\right)=2+2 p(i-2)$ (modulo $\left.2 n\right)$. Since $(n, p)=1$, $\psi$ is a bijection. Similar argument as before shows that $\psi: T_{n, 2, k} \rightarrow T_{2 n, 1,2 p-1}$ is an isomorphism. This proves (a).

Since $G_{4}\left(\operatorname{EG}\left(T_{6,2,2}\right)\right)=3 K_{4}$ and $G_{4}\left(\operatorname{EG}\left(T_{12,1, i}\right)\right)=C_{12}$ for $i=2,3, T_{6,2,2} \not T_{12,1, i}$ for $i=2,3$.

Now, $G_{3}\left(\operatorname{EG}\left(T_{12,1,4}\right)\right)=4 C_{3}$ (with edges $\left.\{i, i+4\}, 1 \leq i \leq 12\right)$. So, $G_{3}\left(\operatorname{EG}\left(T_{12,1,4}\right)\right)$ is a subgraph of $\operatorname{EG}\left(T_{12,1,4}\right)$. Whereas, $G_{3}\left(\operatorname{EG}\left(T_{6,2,2}\right)\right)=4 C_{3}$ (with edges $\left\{u_{i}, u_{j}\right\}$, $\left\{v_{i}, v_{j}\right\}$, where $\left.i-j \equiv 0(\bmod 2)\right)$. So, edges of $G_{3}\left(\operatorname{EG}\left(T_{6,2,2}\right)\right)$ are not in $\operatorname{EG}\left(T_{6,2,2}\right)$. Thus, $T_{6,2,2} ¥ T_{12,1,4}$. This proves (b).
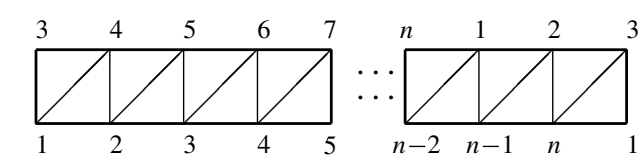

$T_{n, 1,2}$

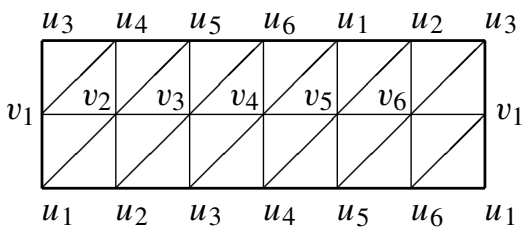

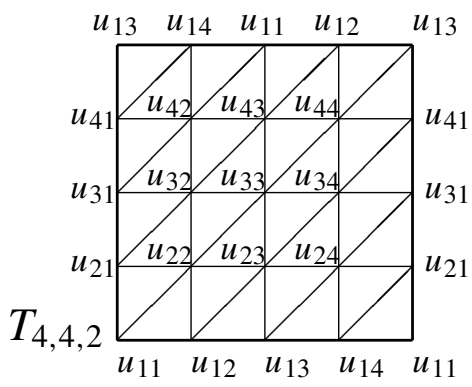


The map $u_{i} \mapsto u_{i}, v_{i} \mapsto v_{i+3}$ defines an isomorphism from $T_{8,2,2}$ to $T_{8,2,4}$. (As usual, addition in the subscripts are modulo 8.) Observe that $G_{1}\left(\operatorname{EG}\left(T_{8,2,2}\right)\right)$ is a null graph, whereas $G_{1}\left(\operatorname{EG}\left(T_{16,1,2}\right)\right)=2 C_{8}, G_{1}\left(\operatorname{EG}\left(T_{16,1,3}\right)\right)=2 C_{8}, G_{1}\left(\operatorname{EG}\left(T_{16,1,6}\right)\right)=4 C_{4}$. Thus $T_{8,2,2} ¥ T_{16,1, j}$ for $j=2,3$ and 6 . Therefore, by Lemma $2.1(\mathrm{~g}), T_{8,2,2} \neq T_{16,1, i}$ for all $i$. This proves (c).

Example 3. Some more weakly regular orientable combinatorial 2-manifolds of Euler characteristic 0 . For $n, m \geq 3$ and $k=0, \ldots, n-1$,

$$
\begin{aligned}
T_{n, m, k}= & \left\{u_{i, j} u_{i, j+1} u_{i+1, j+1}, u_{i, j} u_{i+1, j} u_{i+1, j+1}: 1 \leq i \leq m-1,1 \leq j \leq n\right\} \\
& \cup\left\{u_{m, j} u_{m, j+1} u_{1, j+k+1}, u_{m, j} u_{1, j+k} u_{1, j+k+1}: 1 \leq j \leq n\right\},
\end{aligned}
$$

where $V\left(T_{n, m, k}\right)=\left\{u_{i, j}: 1 \leq i \leq m, 1 \leq j \leq n\right\}$. (Addition in the second subscripts are modulo $n$.) Clearly, $T_{n, m, k}$ triangulates the torus and hence it is an orientable combinatorial 2-manifold on $m n$ vertices. Since the degree of each vertex is $6, T_{n, m, k}$ is degree-regular. $\left(T_{n, m, 0}\right.$ was earlier defined in [5] as $A_{m, n}$.) If $\sigma, \gamma: V\left(T_{n, m, k}\right) \rightarrow V\left(T_{n, m, k}\right)$ are the maps given by $\gamma\left(u_{i, j}\right)=u_{i+1, j}$ for $1 \leq i \leq m-1, \gamma\left(u_{m, j}\right)=u_{1, j+k}$ and $\sigma\left(u_{i, j}\right)=u_{i, j+1}$ then $\sigma$ and $\gamma$ are automorphisms of $T_{n, m, k}$ and hence $\langle\sigma, \gamma\rangle$ is a subgroup of $\operatorname{Aut}\left(T_{n, m, k}\right)$. For any vertex $u_{i, j}, \sigma^{j-1} \gamma^{i-1}\left(u_{1,1}\right)=u_{i, j}$. This implies that the action of $\langle\sigma, \gamma\rangle$ is vertextransitive. Thus $T_{n, m, k}$ is weakly regular.

Lemma 2.4. Let $T_{n, m, k}$ be as in Examples 1, 2, 3. Then

(a) If $n$ and $k$ are relatively prime or $n$ and $k+m$ are relatively prime then $T_{n, m, k}$ is isomorphic to $T_{n m, 1, j}$ for some $j$.

(b) $T_{4,4,2} \cong T_{8,2,2}$.

(c) $T_{16,1, k} \neq T_{4,4,0} \neq T_{8,2, j}$ for all $k$ and $j$.

Proof. Since $(n, k)=1$, there exists $p \in\{1, \ldots, n-1\}$ such that $p k \equiv 1(\bmod n)$. Let $\varphi: V\left(T_{n, m, k}\right) \rightarrow\{1, \ldots, m n\}$ be given by $\varphi\left(u_{i, j}\right)=i+m p(j-1)$ (modulo $m n$ ). Since $(n, p)=1, \varphi$ is a bijection. By the similar argument as in the proof of Lemma 2.3, $\varphi: T_{n, m, k} \rightarrow T_{m n, 1, m p}$ is an isomorphism.

Let $(n, k+m)=1$ where $m \geq 3$. Let $p \in\{1, \ldots, n-1\}$ be such that $p(k+m) \equiv 1$ $(\bmod n)$. Let $\psi: V\left(T_{n, 2, k}\right) \rightarrow\{1, \ldots, m n\}$ be given by $\psi\left(u_{i, j}\right)=i+m p(j-i)$ (modulo $m n)$. Since $(n, p)=1, \psi$ is a bijection. Now, $\psi\left(u_{i, j} u_{i, j+1} u_{i+1, j+1}\right)=\{i+m p(j-i), i+$ $m p(j+1-i), i+1+m p(j-i)\}, \psi\left(u_{i, j} u_{i+1, j} u_{i+1, j+1}\right)=\{i+m p(j-i), i+1+m p(j-$ $i-1), i+1+m p(j-i)\}, \psi\left(u_{m, j} u_{m, j+1} u_{1, j+k+1}\right)=\{m+m p(j-m), m+m p(j+$ $1-m), 1+m p(j+k)\}=\{m+m p(j-m), m+m p(j+1-m), 1+m+m p(j-m)\}$, $\psi\left(u_{m, j} u_{1, j+k} u_{1, j+k+1}\right)=\{m+m p(j-m), 1+m p(j+k-1), 1+m p(j+k)\}=$ $\{m+m p(j-m), 1+m+m p(j-m-1), 1+m+m p(j-m)\} \in T_{m n, 1, m p-1}$. (Clearly, if $m n$ is even then $m n / 2-1 \neq m p-1 \neq m n / 2$ and if $m n$ is odd then $m p-1 \neq(m n-1) / 2$.) Thus $\psi: T_{n, m, k} \rightarrow T_{m n, 1, m p-1}$ is an isomorphism. This proves (a).

The map $u_{i, 1} \mapsto u_{i}, u_{i, 3} \mapsto u_{i+4}, u_{i, 2} \mapsto v_{i}$ and $u_{i, 4} \mapsto v_{i+4}$ for $1 \leq i \leq 4$ defines an isomorphism from $T_{4,4,2}$ to $T_{8,2,2}$. This proves (b).

Since $G_{1}\left(\operatorname{EG}\left(T_{16,1,2}\right)\right)=2 C_{8}, G_{1}\left(\operatorname{EG}\left(T_{16,1,3}\right)\right)=2 C_{8}, G_{1}\left(\operatorname{EG}\left(T_{16,1,6}\right)\right)=4 C_{4}$ and $G_{1}\left(\mathrm{EG}\left(T_{4,4,0}\right)\right)$ is a null graph, $T_{4,4,0}$ is not isomorphic to any of $T_{16,1,2}, T_{16,1,3}$ and $\left.T_{16,1,6}\right)$. Again $G_{4}\left(\operatorname{EG}\left(T_{4,4,0}\right)\right)$ is a null graph whereas $G_{4}\left(\operatorname{EG}\left(T_{8,2,2}\right)\right)=8 K_{2}$. Thus $T_{4,4,0} ¥ T_{8,2,2}$. (c) now follows from parts (a), (c) of Lemma 2.3 and part (g) of Lemma 2.1. 
Now, we will present three series of degree-regular triangulations of the Klein bottle. Among these, $B_{m, n}$ were defined earlier in [5]. The smallest among $Q_{m, n}$ 's, namely $Q_{5,2}$, also defined in [5] as $Q$.

Example 4. A series of degree-regular non-orientable combinatorial 2-manifolds of Euler characteristic 0 . For $m, n \geq 3$,

$$
\begin{aligned}
B_{m, n}= & \left\{v_{i, j} v_{i+1, j} v_{i+1, j+1}, v_{i, j} v_{i, j+1} v_{i+1, j+1}: 1 \leq i \leq n, 1 \leq j \leq m-1\right\} \\
& \cup\left\{v_{i, m} v_{n+2-i, 1} v_{n+1-i, 1}, v_{i, m} v_{i+1, m} v_{n+1-i, 1}: 1 \leq i \leq n\right\},
\end{aligned}
$$

where $V\left(B_{m, n}\right)=\left\{v_{i, j}: 1 \leq i \leq n, 1 \leq j \leq m\right\}$. (Addition in the first subscripts are modulo $n$.) Clearly, $B_{m, n}$ triangulates the Klein bottle and hence it is a nonorientable combinatorial 2-manifold. Since the degree of each vertex is $6, B_{m, n}$ is degree-regular.

Example 5. A series of degree-regular non-orientable combinatorial 2-manifolds of Euler characteristic 0 . For $m \geq 3$ and $n \geq 2$,

$$
\begin{aligned}
K_{m, 2 n}= & \left\{v_{i, j} v_{i, j+1} v_{i+1, j}, v_{i, j+1} v_{i+1, j} v_{i+1, j+1}: 1 \leq i \leq n, 1 \leq j \leq m-1\right\} \\
& \cup\left\{v_{i, m} v_{i+1, m} v_{2 n+2-i, 1}, v_{i+1, m} v_{2 n+2-i, 1} v_{2 n+1-i, 1}: 1 \leq i \leq n\right\} \\
& \cup\left\{v_{i, j} v_{i+1, j} v_{i+1, j+1}, v_{i, j} v_{i, j+1} v_{i+1, j+1}: n+1 \leq i \leq 2 n, 1 \leq j \leq m-1\right\} \\
& \cup\left\{v_{i, m} v_{i+1, m} v_{2 n+1-i, 1}, v_{i, m} v_{2 n+2-i, 1} v_{2 n+1-i, 1}: n+1 \leq i \leq 2 n\right\},
\end{aligned}
$$

where $V\left(K_{m, 2 n}\right)=\left\{v_{i, j}: 1 \leq i \leq 2 n, 1 \leq j \leq m\right\}$. (Addition in the first subscripts are modulo $2 n$.) Clearly, $K_{m, 2 n}$ triangulates the Klein bottle and hence it is a non-orientable combinatorial 2-manifold. Since the degree of each vertex is $6, K_{m, 2 n}$ is degree-regular.
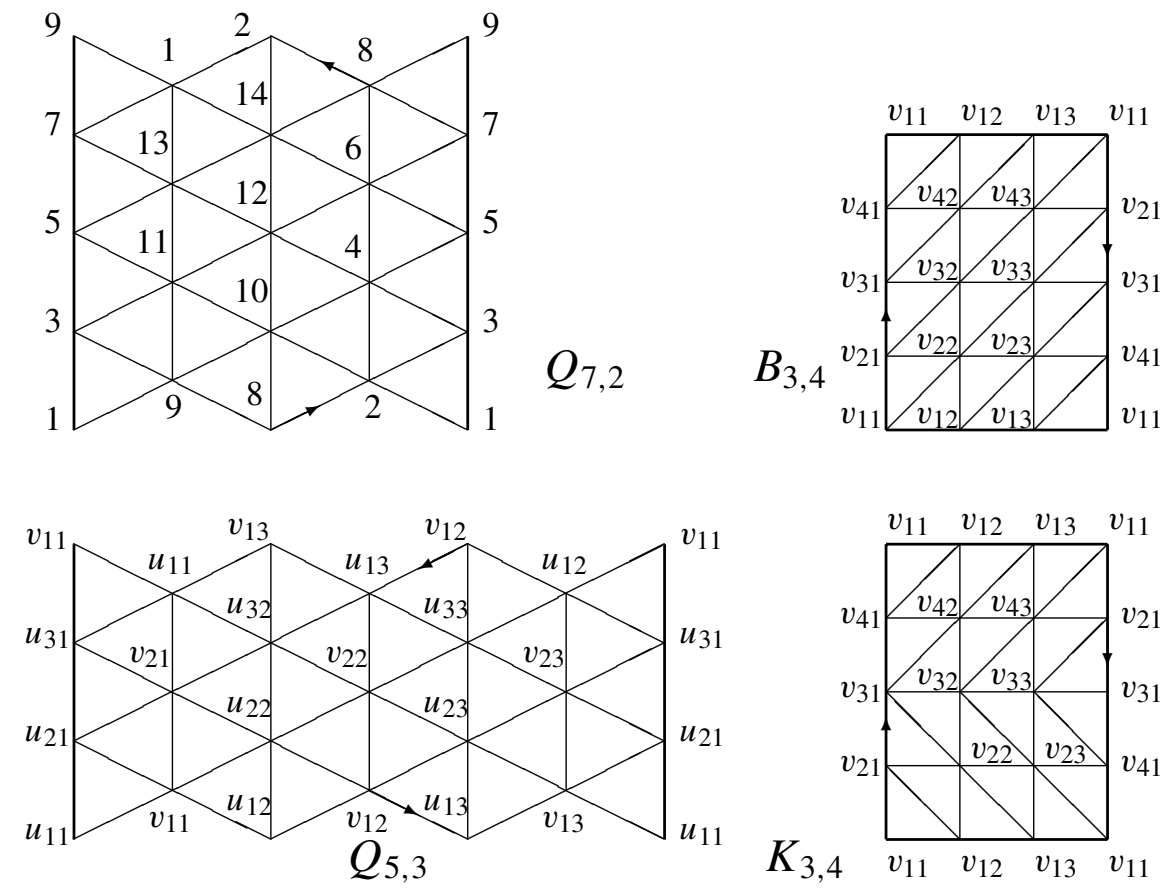
Example 6. A series of weakly regular non-orientable combinatorial 2-manifolds of Euler characteristic 0 . For each $m \geq 2$,

$$
Q_{2 m+1,2}=\{\{i, i+1, i+2\},\{i, i+2, i+2 m+2\}: 1 \leq i \leq 4 m+2\},
$$

where $V\left(Q_{2 m+1,2}\right)=\{1, \ldots, 4 m+2\}$. (Addition modulo $4 m+2$.) Clearly, $Q_{2 m+1,2}$ triangulates the Klein bottle and hence it is a non-orientable combinatorial 2-manifold. Since $\mathbb{Z}_{4 m+2}$ acts transitively (by addition) on vertices, $Q_{2 m+1,2}$ is weakly regular.

More generally, for each $n \geq 2$ we define the following.

Example 7. A series of degree-regular non-orientable combinatorial 2-manifolds of Euler characteristic 0 . For $m, n \geq 2$,

$$
\begin{aligned}
Q_{2 m+1, n}= & \left\{u_{i, j} u_{i+1, j} v_{i, j}, u_{i, j+1} u_{i+1, j+1} v_{i, j}: 1 \leq i \leq m, 1 \leq j \leq n\right\} \\
& \cup\left\{v_{i, j} v_{i+1, j} u_{i+1, j}, v_{i, j} v_{i+1, j} u_{i+1, j+1}: 1 \leq i \leq m-1,1 \leq j \leq n\right\} \\
& \cup\left\{u_{m+1, j} u_{1, n+2-j} v_{1, n+2-j}, u_{m+1, j+1} u_{1, n+2-j} v_{1, n+1-j},\right. \\
& \left.u_{m+1, j} u_{1, n+2-j} v_{m, j}, u_{m+1, j+1} u_{1, n+2-j} v_{m, j}: 1 \leq j \leq n\right\},
\end{aligned}
$$

where $V\left(Q_{2 m+1, n}\right)=\left\{u_{i, j}: 1 \leq i \leq m+1,1 \leq j \leq n\right\} \cup\left\{v_{i, j}: 1 \leq i \leq m, 1 \leq j \leq n\right\}$. (Addition in the second subscripts are modulo $n$.) Clearly, $Q_{2 m+1, n}$ triangulates the Klein bottle and hence it is a non-orientable combinatorial 2-manifold. Since the degree of each vertex is $6, Q_{2 m+1, n}$ is degree-regular.

For $n \geq 3$ there are two induced 3-cycles (induced subcomplexes which are 3-cycles) through $v_{2,1}$ in $Q_{5, n}$, namely, $C_{3}\left(v_{2,1}, u_{1,1}, u_{2,1}\right)$ and $C_{3}\left(v_{2,1}, u_{3,1}, v_{1,1}\right)$. But there is no induced 3-cycle through $v_{2, n}$. So, there does not exist any automorphism of $Q_{5, n}$ which sends $v_{2,1}$ to $v_{2, n}$. Thus, $Q_{5, n}$ is not weakly regular for $n \geq 3$.

Observe that $G_{3}\left(\operatorname{EG}\left(Q_{7,2 t-1}\right)\right)=C_{7}\left(u_{1,1}, u_{3,1}, v_{1,1}, v_{3,1}, u_{2,1}, u_{4,1}, v_{2,1}\right) \sqcup C_{7}\left(u_{1, t+1}\right.$, $\left.u_{3, t+1}, v_{1, t}, v_{3, t}, u_{2, t+1}, u_{4, t+1}, v_{2, t}\right)$ and $G_{3}\left(\operatorname{EG}\left(Q_{7,2 t-2}\right)\right)=C_{7}\left(u_{1,1}, u_{3,1}, v_{1,1}, v_{3,1}\right.$, $\left.u_{2,1}, u_{4,1}, v_{2,1}\right) \sqcup C_{7}\left(u_{1, t}, u_{3, t}, v_{1, t}, v_{3, t}, u_{2, t}, u_{4, t}, v_{2, t}\right)$ for $t \geq 2$. So, for $n \geq 2$, $G_{3}\left(\operatorname{EG}\left(Q_{7, n}\right)\right)$ consists of two disjoint 7-cycles. This implies that $G_{3}\left(\operatorname{EG}\left(Q_{7, n}\right)\right)$ is not regular for $n \geq 3$ and hence $Q_{7, n}$ is not weakly regular for $n \geq 3$.

For each $m \geq 2$, there are exactly two $(2 m+1)$-cycles (namely, $C_{2 m+1}\left(u_{1,1}, \ldots, u_{m+1,1}\right.$, $\left.v_{1,1}, \ldots, v_{m, 1}\right), C_{2 m+1}\left(u_{1, t+1}, \ldots, u_{m+1, t+1}, v_{1, t}, \ldots, v_{m, t}\right)$ in $Q_{2 m+1,2 t-1}$ and $C_{2 m+1}$ $\left(u_{1,1}, \ldots, u_{m+1,1}, v_{1,1}, \ldots, v_{m, 1}\right), C_{2 m+1}\left(u_{1, t+1}, \ldots, u_{m+1, t+1}, v_{1, t+1}, \ldots, v_{m, t+1}\right)$ in $\left.Q_{2 m+1,2 t}\right)$ each of which is the boundary of a $(2 m+1)$-vertex Möbius strip. In other words, there are exactly two $(2 m+1)$-cycle, say $C_{1}$ and $C_{2}$, such that $\left|Q_{2 m-1, n}\right| \backslash\left|C_{i}\right|$ is the union of two disjoint open Möbius strips for $i=1,2$. Thus, if $n \geq 3$ and $u$ is a vertex in $C_{1}$ and $v$ is a vertex outside $C_{1} \cup C_{2}$ then there does not exist any automorphism of $Q_{2 m+1, n}$ which sends $u$ to $v$. So, $Q_{2 m+1, n}$ is not weakly regular for all $m \geq 2$ and $n \geq 3$.

Lemma 2.5. Let $B_{m, n}, K_{m, 2 n}$ and $Q_{2 m+1, n}$ be as above. We have the following:

(a) $B_{3,4} \neq B_{4,3} \approx K_{3,4} \approx B_{3,4}$.

(b) $B_{3,5} \neq B_{5,3} \neq Q_{5,3} \neq B_{3,5}$.

(c) None of $B_{3,4}, B_{4,3}, K_{3,4}, B_{3,5}, B_{5,3}, Q_{5,3}$ are weakly regular. 
Proof. Observe that $G_{4}\left(\mathrm{EG}\left(B_{3,4}\right)\right)=C_{3}\left(v_{11}, v_{42}, v_{23}\right) \cup C_{3}\left(v_{41}, v_{12}, v_{13}\right) \cup C_{3}\left(v_{31}, v_{22}\right.$, $\left.v_{43}\right) \cup C_{3}\left(v_{21}, v_{32}, v_{33}\right), G_{4}\left(\operatorname{EG}\left(B_{4,3}\right)\right)=C_{8}\left(v_{11}, v_{32}, v_{23}, v_{14}, v_{21}, v_{12}, v_{33}, v_{24}\right) \cup$ $K_{4}\left(\left\{v_{31}, v_{22}, v_{13}, v_{34}\right\}\right)$ and $G_{4}\left(\operatorname{EG}\left(K_{3,4}\right)\right)=K_{4}\left(\left\{v_{11}, v_{22}, v_{33}, v_{42}\right\}\right) \cup K_{4}\left(\left\{v_{12}, v_{23}, v_{31}\right.\right.$, $\left.\left.v_{43}\right\}\right) \cup K_{4}\left(\left\{v_{13}, v_{21}, v_{32}, v_{41}\right\}\right)$. These prove (a) (since $M \cong N$ implies $G_{4}(\mathrm{EG}(M)) \cong$ $\left.G_{4}(\mathrm{EG}(N))\right)$.

It is easy to see the following: (i) $G_{4}\left(\mathrm{EG}\left(B_{5,3}\right)\right)=C_{5}\left(v_{21}, v_{12}, v_{33}, v_{24}, v_{15}\right) \cup$ $C_{10}\left(v_{11}, v_{32}, v_{23}, v_{14}, v_{35}, v_{31}, v_{22}, v_{13}, v_{34}, v_{25}\right)$, (ii) $G_{4}\left(\mathrm{EG}\left(B_{3,5}\right)\right)$ is $C_{3}\left(v_{11}, v_{52}, v_{23}\right)$ $\cup C_{3}\left(v_{31}, v_{32}, v_{43}\right) \cup C_{3}\left(v_{21}, v_{42}, v_{33}\right) \cup C_{3}\left(v_{51}, v_{12}, v_{13}\right)$ together with the three isolated vertices $v_{41}, v_{22}, v_{53}$ and (iii) $G_{4}\left(\operatorname{EG}\left(Q_{5,3}\right)\right)$ is $C_{5}\left(u_{11}, u_{21}, u_{31}, v_{11}, v_{21}\right) \cup$ $C_{5}\left(u_{13}, u_{23}, u_{33}, v_{12}, v_{22}\right)$ together with five isolated vertices. These prove (b).

If $M$ is weakly regular then $\operatorname{Aut}(M)$ acts vertex-transitively on $G_{n}(\mathrm{EG}(M))$ for all $n \geq 0$. Since $G_{4}\left(\operatorname{EG}\left(B_{4,3}\right)\right)=C_{8} \sqcup K_{4}$, no group can act vertex-transitively on $G_{4}\left(\operatorname{EG}\left(B_{4,3}\right)\right)$. So, $B_{4,3}$ is not weakly regular. Similarly, $B_{5,3}$ is not weakly regular. Since $G_{4}\left(\operatorname{EG}\left(B_{3,5}\right)\right)$ and $G_{4}\left(\mathrm{EG}\left(Q_{5,3}\right)\right)$ are not regular graphs, $B_{3,5}$ and $Q_{5,3}$ are not weakly regular.

Observe that $G_{3}\left(\operatorname{EG}\left(B_{3,4}\right)\right) \cap \operatorname{EG}\left(B_{3,4}\right)=C_{6}\left(v_{11}, v_{12}, v_{23}, v_{41}, v_{42}, v_{13}\right) \cup C_{6}\left(v_{31}\right.$, $\left.v_{32}, v_{43}, v_{21}, v_{22}, v_{33}\right)$. If possible let there be $\sigma \in \operatorname{Aut}\left(B_{3,4}\right)$ such that $\sigma\left(v_{11}\right)=v_{12}$. Since Aut $\left(B_{3,4}\right)$ acts vertex-transitively on the graph $G_{3}\left(\operatorname{EG}\left(B_{3,4}\right)\right) \cap \operatorname{EG}\left(B_{3,4}\right), \sigma\left(v_{12}\right)=v_{11}$ or $v_{23}$. In the first case, $\sigma\left(v_{41}\right)=v_{42}$ and hence $\sigma\left(v_{11} v_{12} v_{41}\right)=v_{11} v_{12} v_{42}$. But $v_{11} v_{12} v_{41}$ is a face, whereas $v_{11} v_{12} v_{42}$ is not a face, a contradiction. In the second case, $\left(v_{11}, v_{12}, v_{23}, v_{41}\right.$, $\left.v_{42}, v_{13}\right)$ is a cycle in (the permutation) $\sigma$. Then $\sigma\left(v_{11} v_{12} v_{41}\right)=v_{12} v_{23} v_{42}$. But $v_{11} v_{12} v_{41}$ is a face, whereas $v_{12} v_{23} v_{42}$ is not a face, a contradiction. So, there is no automorphism which maps $v_{11}$ to $v_{12}$. Therefore, $B_{3,4}$ is not weakly regular.

If possible let there be $\tau \in \operatorname{Aut}\left(K_{3,4}\right)$ such that $\tau\left(v_{11}\right)=v_{21}$. Since $G_{3}\left(\operatorname{EG}\left(K_{3,4}\right)\right)=$ $C_{3}\left(v_{11}, v_{12}, v_{13}\right) \cup C_{3}\left(v_{21}, v_{23}, v_{42}\right) \cup C_{3}\left(v_{31}, v_{32}, v_{33}\right) \cup C_{3}\left(v_{41}, v_{22}, v_{43}\right), \tau\left(v_{12}\right)=v_{23}$ or $v_{42}$. In either case $\tau$ maps the edge $v_{11} v_{12}$ of $K_{3,4}$ to a non-edge of $K_{3,4}$, a contradiction. So, there is no automorphism which maps $v_{11}$ to $v_{21}$. Thus, $K_{3,4}$ is not weakly regular.

Example 8. A triangulation $(E)$ of the plane $\mathbb{R}^{2}$. The vertex-set $V(E)=\left\{u_{m, 2 n}=\right.$ $\left.(m, n \sqrt{3}), u_{m, 2 n-1}=\left(m+\frac{1}{2}, \frac{(2 n-1) \sqrt{3}}{2}\right): m, n \in \mathbb{Z}\right\}$ and the faces are $\left\{u_{m, 2 n} u_{m+1,2 n}\right.$ $u_{m, 2 n+1}, u_{m+1,2 n} u_{m, 2 n+1} u_{m+1,2 n+1}, u_{m, 2 n-1} u_{m+1,2 n-1} u_{m+1,2 n}, u_{m, 2 n-1} u_{m, 2 n} u_{m+1,2 n}$ : $m, n \in \mathbb{Z}\}$. The group $H$ of translations generated by $\alpha_{1}: u \mapsto u+u_{1,0}$ and $\alpha_{2}: u \mapsto u+u_{0,1}$ is a subgroup of $\operatorname{Aut}(E)$. Clearly, $H$ acts transitively on $V(E)$. The stabilizer of any vertex $u$ in $\operatorname{Aut}(E)$ is isomorphic to the dihedral group $D_{6}$ (of order 12) which acts transitively on the set of flags containing $u$. So, $E$ is combinatorially regular. Let $G_{0}$ denote the stabilizer of $u_{0,0}$. Since $H$ acts transitively on $V(E)$, Aut $(E)=\left\langle H, G_{0}\right\rangle$. This implies that if $\sigma \in \operatorname{Aut}(E)$ has no fixed element in $E$ (vertex, edge or face) then either $\sigma \in H \backslash\{$ Id $\}$ or

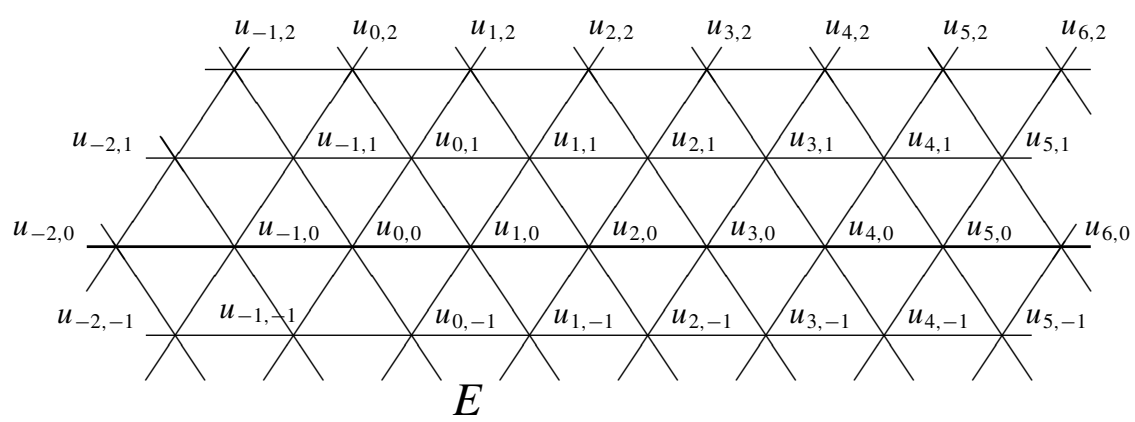


is a glide reflection (i.e., an automorphism of the form $t_{a} \circ r_{l}$, where $r_{l} \in \operatorname{Aut}(E)$ is the reflection about a line $l$ through some vertex and of slope a multiple of $\pi / 6$ and $t_{a} \in H$ is the translation by a nonzero vector $a$ parallel to $l$ ).

\section{Proofs}

Lemma 3.1. There is no triangulation of the closed 2-disk such that (i) the degree of each vertex (except one) in the boundary is 4 and (ii) the degree of each interior vertex is 6.

Proof. If possible let there be a triangulation $K$ of the closed 2-disk on $m+n+1$ vertices with $n$ interior vertices such that the degree of each interior vertex is 6 , the degree of one vertex in the boundary is $k(\geq 2)$ and the degree of each of the remaining $m$ vertices in the boundary is 4 . Then $f_{0}(K)=n+m+1, f_{1}(K)=\frac{6 n+4 m+k}{2}$ and $f_{2}(K)=$ $\frac{6 n+3 m+k-1}{3}$. Therefore, $1=\chi(K)=f_{0}(K)-f_{1}(K)+f_{2}(K)=n+m+1-(3 n+$ $2 m+k / 2)+(2 n+m+(k-1) / 3)$. This implies that $k=-2$, a contradiction. This proves the lemma.

Lemma 3.2. Let $E$ be as in Example 8 and let $M$ be a triangulation of the plane $\mathbb{R}^{2}$. If the degree of each vertex of $M$ is 6 then $M$ is isomorphic to $E$.

Proof. Choose an edge, say $v_{0,0} v_{1,0}$. Then there exists a unique vertex, say $v_{2,0}$, in $\operatorname{lk}\left(v_{1,0}\right)$ such that each side of the segment $v_{0,0} v_{1,0} v_{2,0}$ (union of two line segments) contains three faces from $\operatorname{st}\left(v_{1,0}\right)$ (i.e., $\operatorname{lk}\left(v_{1,0}\right)$ is of the form $\left.C_{6}\left(v_{0,0}, x, y, v_{2,0}, z, w\right)\right)$. Now, given $v_{1,0}$ and $v_{2,0}$ there exists unique vertex $v_{3,0}$ in $\operatorname{lk}\left(v_{2,0}\right)$ such that each side of the segment $v_{1,0} v_{2,0} v_{3,0}$ contains three faces from st $\left(v_{2,0}\right)$. Similarly, given $v_{1,0}$ and $v_{0,0}$ there exists unique vertex $v_{-1,0}$ in $\operatorname{lk}\left(v_{0,0}\right)$ such that each side of the segment $v_{1,0} v_{0,0} v_{-1,0}$ contains three faces from $\operatorname{st}\left(v_{0,0}\right)$. Continuing this way we get vertices $v_{i, 0}, i \in \mathbb{Z}$, such that each side of the segment $v_{i-1,0} v_{i, 0} v_{i+1,0}$ contains three faces from $\operatorname{st}\left(v_{i, 0}\right)$. Because of Lemma 3.1, all these vertices are distinct. So, we get a triangulation of a line (see the figure).

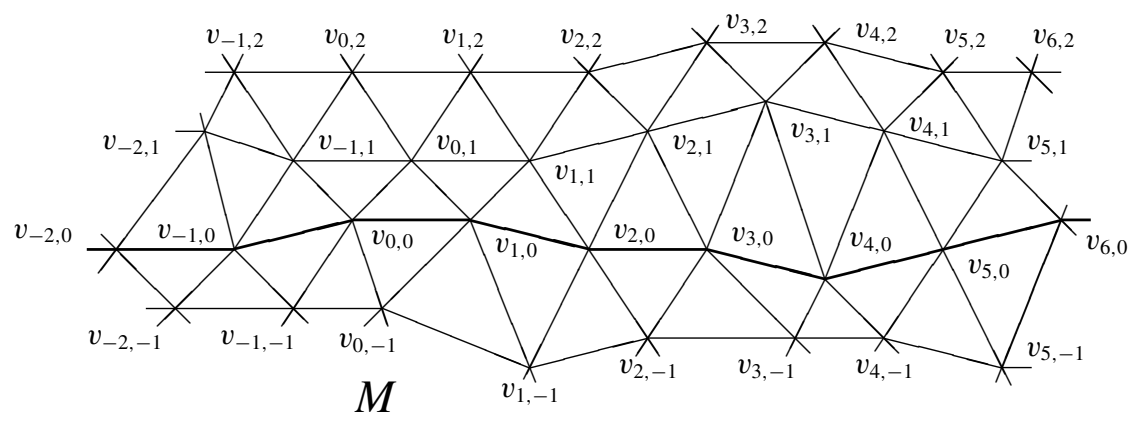

Let $\operatorname{lk}\left(v_{1,0}\right)=C_{6}\left(v_{0,0}, v_{0,1}, v_{1,1}, v_{2,0}, v_{1,-1}, v_{0,-1}\right)$. By the same argument as above, there exists a unique vertex, say $v_{2,1}$, in $\operatorname{lk}\left(v_{1,1}\right)$ such that each side of the segment $v_{0,1} v_{1,1} v_{2,1}$ contains three faces from $\operatorname{st}\left(v_{1,1}\right)$. This implies that $\operatorname{lk}\left(v_{2,0}\right)$ is of the form $C_{6}\left(v_{1,0}, v_{1,1}, v_{2,1}, v_{3,0}, x, v_{1,-1}\right)$. If we continue this way we get vertices $v_{i, 1}, i \in \mathbb{Z}$, such that each side of the segment $v_{i-1,1} v_{i, 1} v_{i+1,1}$ contains three faces from $\operatorname{st}\left(v_{i, 1}\right)$ and $v_{i, 0} v_{i+1,0} v_{i, 1}, v_{i+1,0} v_{i, 1} v_{i+1,1}$ are faces for all $i \in \mathbb{Z}$. 
Similarly, we get: (i) vertices $v_{i, 2}, i \in \mathbb{Z}$, such that each side of the segment $v_{i-1,2} v_{i, 2} v_{i+1,2}$ contains three faces from $\operatorname{st}\left(v_{i, 2}\right)$ and $v_{i, 1} v_{i+1,1} v_{i+1,2}, v_{i, 1} v_{i, 2} v_{i+1,2}$ are faces for all $i \in \mathbb{Z}$, (ii) vertices $v_{i,-1}, i \in \mathbb{Z}$, such that each side of the segment $v_{i-1,-1} v_{i,-1} v_{i+1,-1}$ contains three faces from $\operatorname{st}\left(v_{i,-1}\right)$ and $v_{i,-1} v_{i+1,-1} v_{i+1,0}$, $v_{i,-1} v_{i, 0} v_{i+1,0}$ are faces for all $i \in \mathbb{Z}$.

Continuing this way we get vertices $v_{i, j}, i, j \in \mathbb{Z}$, of $M$ such that each side of the segment $v_{i-1, j} v_{i, j} v_{i+1, j}$ contains three faces from $\operatorname{st}\left(v_{i, j}\right)$ and $v_{i, 2 k} v_{i+1,2 k} v_{i, 2 k+1}$, $v_{i+1,2 k} v_{i, 2 k+1} v_{i+1,2 k+1}, v_{i, 2 k+1} v_{i+1,2 k+1} v_{i+1,2 k+2}, v_{i, 2 k+1} v_{i, 2 k+2} v_{i+1,2 k+2}$ are faces for all $i, j, k \in \mathbb{Z}$. Since $M$ is connected $\left\{v_{i, j}: i, j \in \mathbb{Z}\right\}$ is the vertex-set of $M$. Then $\varphi: V(M) \rightarrow V(E)$, given by $\varphi\left(v_{i, j}\right)=u_{i, j}$, is an isomorphism. This proves the lemma.

Proof of Theorem 1. Let $K$ be a degree-regular triangulation of the torus. Since $\mathbb{R}^{2}$ is the universal cover of the torus, there exists a triangulation $M$ of $\mathbb{R}^{2}$ and a simplicial covering map $\eta: M \rightarrow K$ (see p. 144 of [12]). Since the degree of each vertex in $K$ is 6 , the degree of each vertex in $M$ is 6 . Because of Lemma 3.2, we may assume that $M=E$.

Let $\Gamma$ be the group of covering transformations. Then $|K|=|E| / \Gamma$. For $\sigma \in \Gamma$, $\eta \circ \sigma=\eta$. So, $\sigma$ maps the geometric carrier of a simplex to the geometric carrier of a simplex. This implies that $\sigma$ induces an automorphism $\sigma$ of $E$. Thus, we can identify $\Gamma$ with a subgroup of $\operatorname{Aut}(E)$. So, $K$ is a quotient of $E$ by a subgroup $\Gamma$ of $\operatorname{Aut}(E)$, where $\Gamma$ has no fixed element (vertex, edge or face). Hence $\Gamma$ consists of translations and glide reflections. Since $K=E / \Gamma$ is orientable, $\Gamma$ does not contain any glide reflection. Thus $\Gamma \leq H$ (the group of translations). Now $H$ is commutative. So, $\Gamma$ is a normal subgroup of $H$. Since $H$ acts transitively on $V(E), H / \Gamma$ acts transitively on the vertices of $E / \Gamma$. Thus, $K$ is weakly regular.

Lemma 3.3. For a prime $n \geq 7$, if $M$ is an $n$-vertex weakly regular combinatorial 2manifold of Euler characteristic 0 then $M$ is isomorphic to $T_{n, 1, k}$ for some $k$.

Proof. Since $M$ is weakly regular, it is degree-regular. Let $d$ be the degree of each vertex. Then $n d=2 f_{1}(M)$ and $n-f_{1}(M)+f_{2}(M)=\chi(M)=0$. Since each edge is in two triangles, $2 f_{1}(M)=3 f_{2}(M)$. These imply that $d=6$.

Let $G=\operatorname{Aut}(M)$. Then $G$ is isomorphic to a subgroup of the permutation group $S_{n}$ and hence $G$ is a finite group. Fix a vertex $u$ of $M$. Let $H$ be the stabilizer of $u$ in $G$. Since $M$ is weakly regular, the orbit of $u$ under the action of $G$ contains all the $n$ vertices and hence the index of $H$ in $G$ is $n$. Thus, $n$ divides the order of $G$. Since $n$ is prime, $G$ has an element, say $\tau$, of order $n$.

Let $v$ be a vertex in $M$ such that $\tau(v) \neq v$. Then $V(M)=\left\{v, \tau(v), \ldots, \tau^{n-1}(v)\right\}$. Choose an edge $e$ containing $v$. Let $e=v \tau^{k}(v)$. Then $\sigma=\tau^{k}$ is again an automorphism of order $n$ and $V(M)=\left\{v_{0}=v, v_{1}=\sigma(v), \ldots, v_{n-1}=\sigma^{n-1}(v)\right\}$. For each $i=$ $0, \ldots, n-1, \sigma^{i}$ is an automorphism. Thus, $v_{k} v_{l}$ is an edge implies that $v_{k+i} v_{l+i}$ is an edge and $v_{k} v_{l} v_{j}$ is a face implies that $v_{k+i} v_{l+i} v_{j+i}$ is a face for each $i$. Since $v_{0} v_{1}$ is an edge, $v_{i} v_{i+1}$ is an edge for each $i$. (Addition in the subscripts are modulo $n$.)

Claim. $v_{0} v_{1} v_{2}, v_{0} v_{1} v_{n-1}$ and $v_{0} v_{1} v_{\frac{n+1}{2}}$ are not faces.

If $v_{0} v_{1} v_{2}$ is a face then $v_{n-2} v_{n-1} v_{0}, v_{n-1} v_{0} v_{1}$ are faces. Let $v_{0} v_{2} v_{i}\left(\neq v_{0} v_{1} v_{2}\right)$ be the second face containing $v_{0} v_{2}$. Then $v_{n-2} v_{0} v_{i-2}$ is a face and hence $\operatorname{lk}\left(v_{0}\right)=C_{6}\left(v_{i}\right.$, $\left.v_{2}, v_{1}, v_{n-1}, v_{n-2}, v_{i-2}\right)$. Then $v_{i-2} v_{i} v_{0}$ is a face and hence $v_{0} v_{2} v_{n-i+2}$ is a face. This implies that $n-i+2=1$ or $i$. Since $i \neq 1, n-i+2=i$. Then $n=2 i-2$. This is not possible since $n$ is a prime. So, $v_{0} v_{1} v_{2}$ is not a face and hence $v_{0} v_{1} v_{n-1}$ is not a face. 
Let $c=\frac{n+1}{2}$. If $v_{0} v_{1} v_{c}$ is a face then $v_{n-1} v_{0} v_{c-1}, v_{c-1} v_{c} v_{0}$ are faces. Let $v_{0} v_{1} v_{i}$ $\left(\neq v_{0} v_{1} v_{c}\right.$ ) be the second face containing $v_{0} v_{1}$. Then $v_{n-1} v_{0} v_{i-1}$ is a face and hence $\operatorname{lk}\left(v_{0}\right)=C_{6}\left(v_{i}, v_{1}, v_{c}, v_{c-1}, v_{n-1}, v_{i-1}\right)$. Then $v_{i-1} v_{i} v_{0}$ is a face and hence $v_{0} v_{1} v_{n-i+1}$ is a face. This implies that $n-i+1=c$ or $i$. In either case, we get $i=c$. This is not possible since $v_{0} v_{1} v_{i} \neq v_{0} v_{1} v_{c}$. So, $v_{0} v_{1} v_{c}$ is not a face, where $c=\frac{n+1}{2}$. This proves the claim.

Let $v_{0} v_{1} v_{k}$ be a face containing $v_{0} v_{1}$. Then, by the claim, $k \in\left\{3, \ldots, \frac{n-1}{2}, \frac{n+3}{2}, \ldots, n-\right.$ $2\}$. Now, $v_{0} v_{1} v_{k} \in M$ implies $v_{n-1} v_{0} v_{k-1}, v_{n-k} v_{n-k+1} v_{0} \in M$. Then $V\left(\operatorname{lk}\left(v_{0}\right)\right)=\left\{v_{k-1}\right.$, $\left.v_{n-1}, v_{n-k}, v_{n-k+1}, v_{1}, v_{k}\right\}$ and hence $V\left(\operatorname{lk}\left(v_{1}\right)\right)=\left\{v_{k}, v_{0}, v_{n-k+1}, v_{n-k+2}, v_{2}, v_{k+1}\right\}$. Thus, $V\left(\operatorname{lk}\left(v_{0}\right)\right) \cap V\left(\operatorname{lk}\left(v_{1}\right)\right)=\left\{v_{k}, v_{n-k+1}\right\}$ and hence $v_{0} v_{1} v_{n-k+1} \in M$. This gives $v_{n-1} v_{0} v_{n-k} \in M$. Then $\operatorname{lk}\left(v_{0}\right)=C_{6}\left(v_{k-1}, v_{n-1}, v_{n-k}, v_{n-k+1}, v_{1}, v_{k}\right)$ and hence $\operatorname{lk}\left(v_{i}\right)=$ $C_{6}\left(v_{i+k-1}, v_{n+i-1}, v_{n+i-k}, v_{n+i-k+1}, v_{i+1}, v_{i+k}\right)$ for all $i$. Now, $M \cong T_{n, 1, k-1}$ by the $\operatorname{map} \varphi: V(M) \rightarrow\{1, \ldots, n\}$ given by $\varphi\left(v_{j}\right)=j$ for $1 \leq i \leq n-1$ and $\varphi\left(v_{0}\right)=n$.

Lemma 3.4. Let $G$ be a group of order $24 \times 23$. Then $G$ has a unique (and hence normal) subgroup of order 23.

Proof. Clearly, the number of Sylow 23-subgroups of $G$ is 24 or 1 . If possible let there be 24 Sylow 23-subgroups. Let $H$ be a Sylow 23-subgroup. Let $N(H)$ be the normalizer of $H$ in $G$. Since all the Sylow 23-subgroups are conjugates of $H$ and $|G|=|N(H)| \times$ $\mid$ \{conjugates of $H\}|| N,(H) \mid=23$ and hence $N(H)=H$.

Let $A$ be the set of Sylow 3 -subgroups. Then $H$ acts on $A$ by conjugation. Since there is a Sylow 2-subgroup, the number of elements of order 3 is at most 16 and hence $\#(A)<23$. This implies that the action of $H$ on $A$ is trivial. Let $K \in A$. Then $x K x^{-1}=K$ for all $x \in H$. So, $H$ acts on $K$ by conjugation. Since $|K|=3$, this action of $H$ on $K$ is trivial. So, $x y=y x$ for all $x \in H$ and $y \in K$. This implies that $K \subseteq N(H)$. This is a contradiction since $N(H)=H$. This proves the lemma.

Lemma 3.5. Let $M$ be a connected combinatorial 2-manifold. Then the number of flags in $M$ is divisible by the order of $\operatorname{Aut}(M)$.

Proof. Let $G=\operatorname{Aut}(M)$ and let $\mathcal{F}$ denote the set of flags of $M$. Then $G$ acts on $\mathcal{F}$. Let $\sigma \in G$. If there exists a flag $F=(u, u v, u v w)$ such that $\sigma(F)=F$ then $\sigma(v)=v$ and $\sigma(w)=w$. This implies that $\left.\sigma\right|_{\operatorname{lk}(u)} \equiv \mathrm{Id}$. Since $M$ is connected, this implies that $\sigma \equiv \mathrm{Id}$. Thus, no element of $\mathcal{F}$ is fixed by a non-identity element of $G$. Therefore, the length of each orbit in $\mathcal{F}$ is same as the order of $G$. This proves the lemma.

Lemma 3.6. Let $K$ be a degree-regular triangulation of the torus on $2 p$ vertices. If $p$ is prime and $\geq 13$ then $\operatorname{Aut}(K)$ has a normal subgroup of order $p$.

Proof. Let $G=\operatorname{Aut}(K)$. By Lemma 3.5, $|G|$ is a factor of $2 p \times 6 \times 2=24 p$. By Theorem $1, G$ acts transitively on $V(K)$. So, the index of the stabilizer of a vertex is $2 p$. Thus, $2 p$ (and hence $p$ ) divides the order of $G$. Since $p$ is prime, $G$ has an element, say $\sigma$, of order $p$.

Since $|G|$ is a factor of $24 p$, by Sylow's theorem, $G$ has a unique Sylow $p$-subgroup for $p=13,17,19$ or $p>23$. If $p=23$ and $|G|<24 p$ then, by Sylow's theorem, $G$ has a unique Sylow $p$-subgroup. Finally, if $p=23$ and $|G|=24 \times 23$ then, by Lemma 3.4, $G$ has a unique Sylow 23-subgroup. Therefore, $H=\langle\sigma\rangle$ is the unique (and hence normal) subgroup of order $p$ in $G$. 
Proof of Theorem 2. Let $n \geq 9$ be a composite number. Then either $n=m k$ for some $m, k \geq 3$ or $n=2 p$ for some prime $p \geq 5$. For $m, k \geq 3, B_{m, k}$ (defined in Example 4) is an ( $m k$ )-vertex degree-regular triangulation of the Klein bottle. If $p \geq 5$ is a prime then $Q_{p, 2}$ (defined in Example 6) is a (2p)-vertex degree-regular triangulation of the Klein bottle.

Let $p \geq 13$ be a prime. If possible let there be a $p$-vertex degree-regular triangulation $X$ of the Klein bottle. Since the torus is an orientable double cover of the Klein bottle, there exists a (2p)-vertex degree-regular triangulation $K$ of the torus and a simplicial covering map $\eta: K \rightarrow X$. Then $X$ is a quotient of $K$ by a subgroup $\langle\tau\rangle$ of $\operatorname{Aut}(K)$, where $\tau$ is an automorphism of order 2 without a fixed element (vertex, edge or face). Then $u \tau(u)$ is a non-edge for each $u \in V(K)$. If there exist $u, v \in V(K)$ such that $u v$ and $u \tau(v)$ are edges in $K$ then $\operatorname{deg}_{X}(\eta(v))<6$, a contradiction. So, $u$ and $\tau(u)$ are not adjacent to a common vertex for all $u \in V(K)$.

By Lemma 3.6, there exists a normal subgroup $H \leq \operatorname{Aut}(K)$ of order $p$. Let $H=\langle\sigma\rangle$. Then $\langle\sigma, \tau\rangle=\langle\sigma\rangle\langle\tau\rangle$ is a subgroup of order $2 p$. If $\sigma \circ \tau=\tau \circ \sigma$ then $\langle\sigma\rangle$ acts on $K /\langle\tau\rangle$ non-trivially. This implies that $X$ is weakly regular. But, this is not possible by Lemma 3.3. So, $\sigma \circ \tau \neq \tau \circ \sigma$ and hence $\langle\sigma, \tau\rangle \cong D_{p}$.

Claim 1. No vertex is fixed by $\sigma$.

If possible let $\sigma$ has a fixed vertex. Since $K$ is connected, there is an edge of the form $u v$ such that $\sigma(u)=u$ and $\sigma(v) \neq v$. This implies that $u \sigma^{i}(v)$ is an edge for all $i$. Then $\operatorname{deg}(u) \geq p>6$, a contradiction. This proves the claim.

Claim 2. There exists $w \in V(K)$ and $i \neq 0$ such that $w \sigma^{i}(w)$ is an edge.

By Claim $1, \sigma$ can be written as $\sigma=\left(u, \sigma(u), \ldots, \sigma^{p-1}(u)\right)\left(v, \sigma(v), \ldots, \sigma^{p-1}(v)\right)$ (a permutation on $V(K))$. If $u \sigma^{i}(u)$ is a non-edge for all $i$ then the link of $u$ is of the form $C_{6}\left(\sigma^{i_{1}}(v), \ldots, \sigma^{i_{6}}(v)\right)$. Then $\sigma^{i_{1}}(v) \sigma^{i_{2}}(v)$ is an edge. This implies $v \sigma^{i_{2}-i_{1}}(v)$ is an edge. This proves the claim.

By Claim 2, there exists $i \neq 0$ and $w_{0} \in V(K)$ such that $w_{0} \sigma^{i}\left(w_{0}\right)$ is an edge. Let $\alpha=\sigma^{i}$. Then $w_{0} \alpha\left(w_{0}\right)$ is an edge and hence $\alpha^{j-1}\left(w_{0}\right) \alpha^{j}\left(w_{0}\right)$ is an edge for all $j$. Since $p$ is prime, $\langle\alpha, \tau\rangle=\langle\sigma, \tau\rangle \cong D_{p}$. Then $\alpha^{j} \circ \tau=\tau \circ \alpha^{p-j}$ for all $j$.

Since $p$ is odd there exists $i_{0}$ such that $\tau\left(\alpha^{i_{0}}\left(w_{0}\right)\right) \neq \alpha^{j}\left(w_{0}\right)$ for any $j$. Let $u_{0}=$ $\alpha^{i_{0}}\left(w_{0}\right), v_{0}=\tau\left(u_{0}\right), u_{i}=\alpha^{i}\left(u_{0}\right)$ and $v_{i}=\alpha^{i}\left(v_{0}\right)$ for $1 \leq i \leq p-1$. Therefore, $\alpha=\left(u_{0}, u_{1}, \ldots, u_{p-1}\right)\left(v_{0}, v_{1}, \ldots, v_{p-1}\right)$. Then $\tau\left(u_{i}\right)=\tau\left(\alpha^{i}\left(u_{0}\right)\right)=\alpha^{p-i}\left(\tau\left(u_{0}\right)\right)=$ $\alpha^{p-i}\left(v_{0}\right)=v_{p-i}$ and $\tau\left(v_{i}\right)=\tau\left(\alpha^{i}\left(v_{0}\right)\right)=\alpha^{p-i}\left(\tau\left(v_{0}\right)\right)=\alpha^{p-i}\left(u_{0}\right)=u_{p-i}$.

Since $K$ is connected, there exists an edge of the form $u_{i} v_{j}$ and hence there exists an edge of the form $u_{0} v_{k}$ for some $k \in\{0, \ldots, p-1\}$. If $k$ is odd, let $l=\frac{p-k}{2}$. Then $\alpha^{l}\left(u_{0} v_{k}\right)$ is an edge. But, $\alpha^{l}\left(u_{0} v_{k}\right)=u_{l} v_{k+l}=u_{l} v_{p-l}=u_{l} \tau\left(u_{l}\right)$ is a non-edge, a contradiction. If $k$ is even, let $m=\frac{p-k-1}{2}$. Then $\alpha^{m}\left(u_{0} v_{k}\right)$ is an edge. But, $\alpha^{m}\left(u_{0} v_{k}\right)=u_{m} v_{k+m}=$ $u_{m} v_{p-m-1}=u_{m} \tau\left(u_{m+1}\right)$. This is not possible since $u_{m} u_{m+1}$ is an edge. This proves that there is no $p$-vertex degree regular triangulation of the Klein bottle for a prime $p \geq 13$.

If $n=7,8$ or 11 then, by Proposition 3, there does not exist any $n$-vertex degree regular triangulation of the Klein bottle. This completes the proof.

Proof of Theorem 3. Since $T_{n, 1, k}$ is a weakly regular orientable combinatorial 2-manifold of Euler characteristic 0, Part (a) follows from Part (b) of Lemma 2.1 and Part (b) follows from Parts (b), (c), (i) and (j) of Lemma 2.1. Part (c) follows from Example 6.

Proof of Theorem 4. Follows from Lemma 3.3. 
Proof of Corollary 5. Let $M$ be an $n$-vertex degree-regular combinatorial 2-manifold of Euler characteristic 0 . If $n$ is prime then by Theorem 2, $M$ triangulates the torus and hence, by Theorem $1, M$ is weakly regular. Then, by Theorem $4, M$ is isomorphic to $T_{n, 1, k}$ for some $k$. Now, Part (a) follows from Parts (a), (b), (e) and (h) of Lemma 2.1 and Part (b) follows from Parts (a), (b) and (j) of Lemma 2.1.

Lemma 3.7. Let $M$ be a combinatorial 2-manifold and $a_{1}, \ldots, a_{5}$ be five vertices. If the degree of each vertex is 6 then the number of faces in $\operatorname{st}\left(a_{1}\right) \cup \operatorname{st}\left(a_{2}\right) \cup \operatorname{st}\left(a_{3}\right)$ is $\geq 12$ and the number of faces in $\operatorname{st}\left(a_{1}\right) \cup \cdots \cup \operatorname{st}\left(a_{5}\right)$ is $>12$.

Proof. Let $n$ be the number of faces in st $\left(a_{1}\right) \cup \operatorname{st}\left(a_{2}\right) \cup \operatorname{st}\left(a_{3}\right)$. If $a_{1} a_{2} a_{3}$ is a face in $M$ then clearly $n=13$. If $a_{1} a_{2}, a_{2} a_{3}, a_{1} a_{3}$ are edges in $M$ but $a_{1} a_{2} a_{3}$ is not a face then $n=12$. In the other cases, $n \geq 14$.

Let $m$ be the number of faces in $\operatorname{st}\left(a_{1}\right) \cup \cdots \cup \operatorname{st}\left(a_{5}\right)$. By the above argument, $m \geq 12$ and $m=12$ if and only if the induced subcomplex on a set of any three vertices is a $K_{3}$. So, if $m=12$ then the induced subcomplex of $M$ on $\left\{a_{1}, \ldots, a_{5}\right\}$ is a $K_{5}$ and hence the induced subcomplex of $\operatorname{lk}\left(a_{1}\right)$ on $\left\{a_{2}, a_{3}, a_{4}, a_{5}\right\}$ is a null graph on four vertices. This is not possible since $\operatorname{deg}\left(a_{1}\right)=6$. This proves the lemma.

Lemma 3.8. Let $M$ be an n-vertex connected combinatorial 2-manifold. If the degree of each vertex is 6 and $n>7$ then for any vertex $u$ there exist faces of the form $u a b, v a b$ where uv is a non-edge.

Proof. Let $\operatorname{lk}(u)=C_{6}(1, \ldots, 6)$. Since the degree of each vertex is $6,123, \ldots, 456,561$ are not faces. We want to show that there exists $v \notin\{u, 1, \ldots, 6\}$ such that $12 v, \ldots, 56 v$ or $16 v$ is a face. If not then 124 or 125 is a face. Assume, without loss of generality, that 124 is a face. Then (since $146 \in M \Rightarrow \operatorname{deg}(1)=4$ ) the second face containing 16 is 136. Inductively, 256, 145, 346 and 235 are faces. These imply that $x y$ is an edge for $x \neq y \in U:=\{u, 1, \ldots, 6\}$. Since the degree of each vertex is 6 , for $x \in U$ and $z \notin U$, $x z$ is a non-edge and hence (since $M$ has more than 7 vertices) $M$ is not connected. This completes the proof.

Lemma 3.9. Let $M$ be a connected combinatorial 2-manifold and let $U$ be a set of $m$ vertices of $M$. If the degree of each vertex in $M$ is 6 and $m<f_{0}(M)$ then the number of edges in $M[U]$ is at most $3 m-3$.

Proof. Let $V$ be the vertex-set of $M$. Let $n$ be the number of edges in $M[U]$ and let $k$ be the number of edges of the form $a b$, where $a \in U, b \in V \backslash U$ (i.e., $k$ is the number of connecting edge between $U$ and $V \backslash U)$. Since $M$ is connected and $U \neq V, k \neq 0$.

Now, for any connecting edge $a b$, there exists two faces (with vertices both in $U$ and $V \backslash U$ ) containing $a b$. On the other hand, for each such face there exists exactly two connecting edges. This implies $k \geq 3$. If $k \leq 5$ then clearly all faces containing the connecting edges have to be of the form $a b_{1} b_{2}, \ldots, a b_{k-1} b_{k}, a b_{k} b_{1}$, where $a \in U$ and $b_{1}, \ldots, b_{k} \in V \backslash U$ or $a \in V \backslash U$ and $b_{1}, \ldots, b_{k} \in U$. Then $C_{5}\left(b_{1}, \ldots, b_{k}\right) \subseteq \operatorname{lk}(a)$. This is not possible. So, $k \geq 6$.

Counting two ways the number of pairs of the form $(u, e)$, where $u \in U$ and $e$ is an edge containing $u$, we get $6 m=n \times 2+k$ or $2 n=6 m-k \leq 6 m-6$. This proves the lemma.

Lemma 3.10. If $M$ is a 12-vertex degree-regular combinatorial 2-manifolds of Euler characteristic 0 then $M$ is isomorphic to $T_{12,1,2}, T_{12,1,3}, T_{12,1,4}, T_{6,2,2}, B_{3,4}, B_{4,3}$ or $K_{3,4}$. 
Proof. Let $M$ be a 12-vertex degree regular combinatorial 2-manifold of Euler characteristic 0 . Let the vertex set $V$ of $M$ be $\{0, \ldots, 9, u, v\}$. Let $\varphi: V \rightarrow\{1, \ldots, 12\}$ be given by $\varphi(i)=i$ for $1 \leq i \leq 9, \varphi(0)=10, \varphi(u)=11$ and $\varphi(v)=12$.

Since $\chi(M)=0$, the degree of each vertex is 6 . Assume, without loss of generality, that $\operatorname{lk}(0)=C_{6}(1, \ldots, 6)$. Since the degree of each vertex is $6,123, \ldots, 456,561 \notin M$. Since each component contains at least 7 vertices, $M$ is connected. So, by Lemma 3.8, we may assume that 127 is a face. Then $\operatorname{lk}(1)$ has the form $C_{6}(7,2,0,6, x, y)$, for some $x, y \in V$. It is easy to see that $(x, y)=(3,4),(3,5),(3,8),(4,3),(4,5),(4,8),(8,4),(8,9),(8,3)$, $(8,5)$. The cases $(x, y)=(8,3)$ and $(8,5)$ are isomorphic to the case $(x, y)=(4,8)$ by the map $(0,1)(2,6)(3,4,8)(5,7)$ and $(0,1)(3,7)(4,8,5)$ respectively. So, we need not consider the last two cases.

Claim. $(x, y)=(3,4),(3,8),(4,3),(4,8),(8,4)$ or $(8,9)$.

If $(x, y)=(3,5)$, then $045,056,135,157$ are faces and hence $\operatorname{lk}(5)=C_{6}(4,0,6,3$, 1,7). This implies that $C_{3}(1,5,6) \subseteq 1 \mathrm{k}(3)$. This is not possible.

If $(x, y)=(4,5)$ then $\operatorname{lk}(4)=C_{6}(6,1,5,0,3, z)$, where $z=7,8,9, u$ or $v$. If $z=7$ then, as in the previous case, we get a contradiction. So, we may assume that $1 \mathrm{k}(4)=$ $C_{6}(6,1,5,0,3,8)$ and hence $\operatorname{lk}(6)=C_{6}(8,4,1,0,5, w), \operatorname{lk}(5)=C_{6}(7,1,4,0,6, w)$ for some $w \in V$. It is easy to see that $w=9, u$ or $v$. In any case, we get 15 faces not containing any from $\{9, u, v\} \backslash\{w\}$. This is not possible since $M$ has 24 faces. This proves the claim.

Case 1. $(x, y)=(3,4)$, i.e., $\operatorname{lk}(1)=C_{6}(7,2,0,6,3,4)$. Now, $\operatorname{lk}(3)=C_{6}(6,1,4,0,2, z)$ for some $z \in V$. If $z=5$ then $C_{4}(5,3,1,0) \subseteq \operatorname{lk}(6)$. If $z=7$ then $C_{4}(7,3,0,1) \subseteq$ $\operatorname{lk}(2)$. This implies that $z \in\{8,9, u, v\}$. Assume, without loss of generality, that $\operatorname{lk}(3)=$ $C_{6}(6,1,4,0,2,8)$. Now, $\operatorname{lk}(2)=C_{6}(8,3,0,1,7, w)$, for some $w \in V$. If $w \notin\{9, u, v\}$ then we get 14 faces not containing any of $9, u, v$. This is not possible by Lemma 3.7. So, assume without loss of generality that $z=9$, i.e., $1 \mathrm{k}(2)=C_{6}(8,3,0,1,7,9)$.

Completing successively, we get $\operatorname{lk}(6)=C_{6}(8,3,1,0,5, u), \operatorname{lk}(8)=C_{6}(u, 6,3,2$, $9, v), \operatorname{lk}(4)=C_{6}(7,1,3,0,5, v), \operatorname{lk}(5)=C_{6}(v, 4,0,6, u, 9), \operatorname{lk}(9)=C_{6}(7, u, 5, v, 8,2)$, $\operatorname{lk}(u)=C_{6}(7,9,5,6,8, v)$. Here $M \cong B_{3,4}$ by the map $\varphi_{34} \circ(0,9,3,4,7, u, 2, v)(1,8)$ $(5,6)$, where $\varphi_{34}: V \rightarrow V\left(B_{3,4}\right)$ is given by $\varphi_{34}(i)=v_{1 i}, \varphi_{34}(3+i)=v_{2 i}$, $\varphi_{34}(6+i)=v_{3 i}$, for $1 \leq i \leq 3, \varphi_{34}(0)=v_{41}, \varphi_{34}(u)=v_{42}, \varphi_{34}(v)=v_{43}$.

Case 2. $(x, y)=(3,8)$, i.e., $\operatorname{lk}(1)=C_{6}(7,2,0,6,3,8)$. Now, 023, 034, 136 and 138 are faces in $M$. So, $1 \mathrm{k}(3)=C_{6}(2,0,4,8,1,6)$ or $C_{6}(2,0,4,6,1,8)$. In the first case, $1 \mathrm{k}(2)=$ $C_{6}(6,3,0,1,7, z)$ for some $z \in V$. As in Case $1, z$ is a new vertex, say, 9. Then $1 \mathrm{k}(2)=$ $C_{6}(6,3,0,1,7,9)$ and $\operatorname{lk}(6)=C_{6}(9,2,3,1,0,5)$. This gives 15 faces not containing $u$ or $v$. This is not possible. Thus, $\operatorname{lk}(3)=C_{6}(2,0,4,6,1,8)$. Now, $\operatorname{lk}(6)=C_{6}(4,3,1,0,5, w)$ for some $w \in V$. If $w=2,7$ or 8 , then we get 14 faces not containing any of $9, u, v$. This is not possible by Lemma 3.7. So, assume without loss, that $\operatorname{lk}(6)=C_{6}(4,3,1,0,5,9)$.

Completing successively, we get $\operatorname{lk}(4)=C_{6}(9,6,3,0,5, u), \operatorname{lk}(5)=C_{6}(4,0,6,9$, $v, u), \operatorname{lk}(9)=C_{6}(4,6,5, v, 7, u), \operatorname{lk}(7)=C_{6}(9, u, 8,1,2, v), \operatorname{lk}(2)=C_{6}(7,1,0,3$, $8, v), \operatorname{lk}(8)=C_{6}(2,3,1,7, u, v)$. Here $M \cong T_{12,1,2}$ by the $\operatorname{map} \varphi \circ(0,4,6,5,7, u, 9,8, v)$ $(1,2)$.

Case 3. $(x, y)=(4,3)$, i.e., $\operatorname{lk}(1)=C_{6}(7,2,0,6,4,3)$. Now, $\operatorname{lk}(4)=C_{6}(6,1,3,0,5, z)$ for some $z \in V$. If $z=2$, then $\operatorname{lk}(2)$ has 7 vertices. If $z=7$, then $\operatorname{lk}(4)=$ $C_{6}(6,1,3,0,5,7)$ and hence $127,137,457,467$ are faces in $M$. This implies that 
$\operatorname{lk}(7)=C_{6}(2,1,3,5,4,6)$ or $C_{6}(2,1,3,6,4,5)$. In either cases we get 14 faces not containing any of 8,9,u or $v$. This is not possible by Lemma 3.7. This implies that $z=8,9, u$ or $v$. Assume, without loss, that $z=8$. This case is now isomorphic to Case 2 by the map $(0,3,6,2,4,1)(5,8,7)(9, v)$.

Case 4. $(x, y)=(4,8)$, i.e., $\operatorname{lk}(1)=C_{6}(6,0,2,7,8,4)$. This gives $1 \mathrm{k}(4)=C_{6}(8,1$, $6,3,0,5)$.

Now, $\operatorname{lk}(6)=C_{6}(3,4,1,0,5, z)$ for some $z \in V$. By using Lemma 3.7, $z=9, u$ or $v$. So, assume that $\operatorname{lk}(6)=C_{6}(3,4,1,0,5,9)$. This implies that $\operatorname{lk}(5)=C_{6}(9,6,0,4,8, u)$. This case is now isomorphic to Case 1 by the map $(1,6)(2,5)(3,4)(9,7, u)$.

Case 5. $(x, y)=(8,4)$, i.e., $1 \mathrm{k}(1)=C_{6}(6,0,2,7,4,8)$. Now, 034, 045, 147 and 148 are faces in $M$. So, $1 \mathrm{k}(4)=C_{6}(3,0,5,7,1,8)$ or $C_{6}(3,0,5,8,1,7)$.

Subcase 5.1. $\operatorname{lk}(4)=C_{6}(3,0,5,7,1,8)$. Then (by using Lemma 3.7) $\operatorname{lk}(7)=C_{6}(5,4$, $1,2, z, w)$, where $z, w \in\{9, u, v\}$. So, assume without loss, that $\operatorname{lk}(7)=C_{6}(5,4,1,2,9, u)$ Completing successively, we get $\operatorname{lk}(2)=C_{6}(9,7,1,0,3, v), \operatorname{lk}(3)=C_{6}(2,0,4,8, u, v)$, $\operatorname{lk}(u)=C_{6}(8,9,7,5, v, 3), \operatorname{lk}(8)=C_{6}(9, u, 3,4,1,6), \operatorname{lk}(5)=C_{6}(7,4,0,6, v, u)$, $\operatorname{lk}(6)=C_{6}(5,0,1,8,9, v)$. Here $M \cong K_{3,4}$ by the map $\psi_{34} \circ(0,8)(1,9)(2, v, 3, u)(4,7)$, where $\psi_{34}$ is same as $\varphi_{34}$ (of Case 1 ) on the vertex-set.

Subcase 5.2. $\operatorname{lk}(4)=C_{6}(3,0,5,8,1,7)$. Then $\operatorname{lk}(7)=C_{6}(3,4,1,2, z, w)$, for some $z, w \in V$. As in the previous case, $z, w \in\{9, u, v\}$. So, assume without loss, that $\operatorname{lk}(7)=$ $C_{6}(3,4,1,2,9, u)$. Then $\operatorname{lk}(2)=C_{6}(9,7,1,0,3, a)$ for some $a \in V$. It is easy to see that $a=8$ or $v$. If $a=8$ then, considering $\mathrm{lk}(8)$, we get 19 faces not containing $v$. This is not possible since $f_{2}(M)=24$. So, $\operatorname{lk}(2)=C_{6}(9,7,1,0,3, v)$ and hence $\operatorname{lk}(3)=$ $C_{6}(2,0,4,7, u, v)$. Then, $\operatorname{lk}(8)=C_{6}(5,4,1,6, b, c)$, where $b, c \in\{9, u, v\}$. Since the set of known faces is invariant under $(1,4)(2,3)(5,6)(9, u)$, we may assume that $(b, c)=$ $(9, u),(u, 9),(v, u)$ or $(v, 9)$.

Subcase 5.2.1. $\operatorname{lk}(8)=C_{6}(5,4,1,6,9, u)$. Completing successively, we get $\operatorname{lk}(9)=C_{6}(2$, $7, u, 8,6, v), \operatorname{lk}(6)=C_{6}(9,8,1,0,5, v), \operatorname{lk}(5)=C_{6}(6,0,4,8, u, v)$. Here $M \cong T_{12,1,4}$ by the map $\varphi \circ(0,2,4, u, 9,6)(1, v, 8)(3,5,7)$.

Subcase 5.2.2. $\operatorname{lk}(8)=C_{6}(5,4,1,6, u, 9)$. Completing successively, we get $\operatorname{lk}(u)=C_{6}(6$, $8,9,7,3, v), \operatorname{lk}(9)=C_{6}(5,8, u, 7,2, v), \operatorname{lk}(v)=C_{6}(6, u, 3,2,9,5)$. Here $M \cong B_{4,3}$ by the map $\varphi_{43} \circ(0,9, u, 7,6,8, v, 3,2)(1,5,4)$, where $\varphi_{43}: V \rightarrow V\left(B_{4,3}\right)$ is given by $\varphi_{43}(i)=v_{1 i}, \varphi_{43}(4+i)=v_{2 i}$, for $1 \leq i \leq 4, \varphi_{43}(9)=v_{31}, \varphi_{43}(0)=v_{32}, \varphi_{43}(u)=v_{33}$, $\varphi_{43}(v)=v_{34}$.

Subcase 5.2.3. $\operatorname{lk}(8)=C_{6}(5,4,1,6, v, 9)$. Completing successively, we get $\operatorname{lk}(v)=$ $C_{6}(3,2,9,8,6, u), \operatorname{lk}(9)=C_{6}(8, v, 2,7, u, 5), \operatorname{lk}(u)=C_{6}(6,5,9,7,3, v)$. Here $M \cong$ $T_{12,1,3}$ by the map $\varphi \circ(1,0,8,6)(2,5, u, 4,3, v, 9)$.

Subcase 5.2.4. $\operatorname{lk}(8)=C_{6}(5,4,1,6, v, u)$. Completing successively, we get $\operatorname{lk}(v)=C_{6}(3$, $2,9,6,8, u), \operatorname{lk}(u)=C_{6}(3,7,9,5,8, v), \operatorname{lk}(9)=C_{6}(6,5, u, 7,2, v)$. Here $M \cong B_{4,3}$ by the map $\varphi_{43} \circ(1,2,9,4,6,3,5, u, v, 8,7)$, where $\varphi_{43}$ is as in Subcase 5.2.2.

Case 6. $(x, y)=(8,9)$, i.e., $\operatorname{lk}(1)=C_{6}(6,0,2,7,9,8)$. Now, $\operatorname{lk}(2)=C_{6}(7,1,0,3, z, w)$ for some $z, w \in V$. It is easy to see that $(z, w)=(5,6),(6,8),(5,8),(5, u)$, 
$(u, 8),(6,5),(8,6),(8, u),(u, 5),(9, u),(u, 4),(u, v)$. If $(z, w)=(5,8)$, i.e., $\operatorname{lk}(2)=C_{6}(7,1,0,3,5,8)$ then $045,056,235,258$ are faces in $M$. This implies that $\operatorname{lk}(5)=C_{6}(6,0,4,8,2,3)$. Then $\operatorname{deg}(8) \geq 7$. Since the set of known faces are invariant under $(0,1)(3,7)(4,9)(5,8)$, we may assume that $(z, w)=(5,6),(5, u),(6,5),(u, 5)$, $(9, u)$ or $(u, v)$.

Subcase 6.1. $\operatorname{lk}(2)=C_{6}(7,1,0,3,5,6)$. Now, it is easy to see that $\operatorname{lk}(6)=C_{6}(7,2,5,0$, 1, 8). Now, completing successively, we get $\operatorname{lk}(7)=C_{6}(8,6,2,1,9, u), \operatorname{lk}(8)=C_{6}(u, 7$, $6,1,9, v), \operatorname{lk}(9)=C_{6}(8,1,7, u, 4, v), \operatorname{lk}(4)=C_{6}(3,0,5, v, 9, u), \operatorname{lk}(5)=C_{6}(4,0,6,2$, $3, v), \operatorname{lk}(3)=C_{6}(5,2,0,4, u, v)$. Here $M \cong T_{12,1,2}$ by the $\operatorname{map} \varphi \circ(0,9,3, u, 2,8,4, v, 1$, $6,7,5)$.

Subcase 6.2. $\operatorname{lk}(2)=C_{6}(7,1,0,3,5, u)$. Now, it is easy to see that $1 \mathrm{k}(5)=C_{6}(4,0,6,3$, $2, u)$. Now, completing successively, we get $\operatorname{lk}(3)=C_{6}(2,0,4, v, 6,5), \operatorname{lk}(4)=C_{6}(3,0$, $5, u, 9, v), \operatorname{lk}(9)=C_{6}(8,1,7, v, 4, u), \operatorname{lk}(7)=C_{6}(9,1,2, u, 8, v), \operatorname{lk}(8)=C_{6}(1,6, v, 7$, $u, 9), \operatorname{lk}(u)=C_{6}(2,5,4,9,8,7)$. Here $M \cong B_{3,4}$ by the map $\varphi_{34} \circ(0,9,3,4, v, 1,6,5,8$, $2,7)$, where $\varphi_{34}$ is as in Case 1 .

Subcase 6.3. $\operatorname{lk}(2)=C_{6}(7,1,0,3,6,5)$. Completing successively, we get $1 \mathrm{k}(6)=C_{6}(5$, $0,1,8,3,2), \operatorname{lk}(3)=C_{6}(2,0,4, u, 8,6), \operatorname{lk}(5)=C_{6}(6,0,4, v, 7,2), \operatorname{lk}(8)=C_{6}(3,6$, $1,9, v, u), \operatorname{lk}(7)=C_{6}(2,1,9, u, v, 5), \operatorname{lk}(4)=C_{6}(5,0,3, u, 9, v), \operatorname{lk}(u)=C_{6}(4,3,8, v$, $7,9)$. Here $M \cong B_{3,4}$ by the map $\varphi_{34} \circ(0,9,3,5,7, u, 2,8,1, v)(4,6)$, where $\varphi_{34}$ is as in Case 1.

Subcase 6.4. $\operatorname{lk}(2)=C_{6}(7,1,0,3,9, u)$. Completing successively, we get $1 \mathrm{k}(9)=C_{6}(8$, $1,7,3,2, u), \operatorname{lk}(3)=C_{6}(2,0,4, v, 7,9), \operatorname{lk}(7)=C_{6}(3,9,1,2, u, v), \operatorname{lk}(u)=C_{6}(7,2$, $9,8,5, v), \operatorname{lk}(8)=C_{6}(5, u, 9,1,6,4), \operatorname{lk}(5)=C_{6}(8,4,0,6, v, u), \operatorname{lk}(6)=C_{6}(5,0,1,8$, $4, v)$. Here $M \cong B_{3,4}$ by the map $\varphi_{34} \circ(0,7,2)(1, u)(3,6,8, v, 5,4,9)$, where $\varphi_{34}$ is as in Case 1.

Subcase $6.5 . \operatorname{lk}(2)=C_{6}(7,1,0,3, u, 5)$. Now, it is easy to see that $\operatorname{lk}(5)=C_{6}(4,0,6, u$, $2,7)$ or $C_{6}(4,0,6,7,2, u)$. The first case is isomorphic to Subcase 5.1 by the map $(0,1,7,5)(2,4,6)(3,8, u)$. The second case is isomorphic to Subcase 5.2 by the map $(0,4,5)(1,7,2)(3,8, u, 6)$.

Subcase 6.6. $\operatorname{lk}(2)=C_{6}(7,1,0,3, u, v)$. Now, it is easy to see that $\operatorname{lk}(7)=C_{7}(v, 2,1,9$, $a, b)$, where $(a, b)=(3,4),(4,3),(4,5),(4,8),(5,4),(5,6),(5,8),(6,5),(6,8)$, $(u, 3),(u, 4),(u, 5),(u, 8)$. Since the set of known faces is invariant under the map $(1,2)(3,6)(4,5)(8, u)(9, v)$, we may assume that $(a, b)=(3,4),(4,3),(4,5),(4,8)$, $(5,4),(5,8),(6,8),(u, 8)$.

Claim. $(a, b)=(3,4)$ or $(5,4)$.

If $(a, b)=(4,3)$ then, considering $\mathrm{lk}(3)$, we get $C_{4}(u, 3,7,2) \subseteq \mathrm{lk}(v)$. If $(a, b)=$ $(4,5)$ then, $\operatorname{lk}(5)$ can not be a 6-cycle. If $(a, b)=(4,8)$ then, considering $\operatorname{lk}(9)$, we see that $0,1,4,7,8,9 \notin \mathrm{lk}(u)$. This is not possible. If $(a, b)=(5,8)$ then, considering $\operatorname{lk}(8)$, we get $C_{4}(5,8,1,0) \subseteq \mathrm{k}(6)$. If $(a, b)=(6,8)$ then, considering the links of 6,9 and 8 successively, we get $C_{4}(u, 8,7,2) \subseteq \operatorname{lk}(v)$. If $(a, b)=(u, 8)$ then, considering $\mathrm{lk}(u)$, we get 7 vertices in $1 \mathrm{k}(8)$. These prove the claim.

Subcase 6.6.1. $\operatorname{lk}(7)=C_{6}(v, 2,1,9,3,4)$. Completing successively, we get $1 \mathrm{k}(3)$ $=C_{6}(0,2, u, 9,7,4), \operatorname{lk}(9)=C_{6}(1,7,3, u, 5,8), \operatorname{lk}(4)=C_{6}(0,3,7, v, 8,5)$, 
$\operatorname{lk}(5)=C_{6}(0,4,8,9, u, 6), \operatorname{lk}(u)=C_{6}(2,3,9,5,6, v), \operatorname{lk}(6)=C_{6}(0,1,8, v, u, 5)$. Now, $M \cong K_{3,4}$ by the map $\psi_{34} \circ(0,4,5,7,3,2,1, v, 6,9, u)$, where $\psi_{34}$ is as in Subcase 5.1.

Subcase 6.6.2. $\operatorname{lk}(7)=C_{6}(v, 2,1,9,5,4)$. Now, it is easy to see that $\operatorname{lk}(4)=C_{6}(3,0,5,7$, $v, 8)$. Now, $0,1,4,7,8 \notin \operatorname{lk}(u)$. So, $5 \in \operatorname{lk}(u)$ and hence $\operatorname{lk}(5)=C_{6}(6,0,4,7,9, u)$. Then $\operatorname{lk}(3)=C_{6}(8,4,0,2, u, 6)$ or $C_{6}(8,4,0,2, u, 9)$.

Subcase 6.6.2.1. $\operatorname{lk}(3)=C_{6}(8,4,0,2, u, 6)$. Completing successively, we get $1 \mathrm{k}(6)=$ $C_{6}(0,1,8,3, u, 5), \operatorname{lk}(8)=C_{6}(1,6,3,4, v, 9), \operatorname{lk}(9)=C_{6}(1,7,5, u, v, 8)$. Now, $M \cong K_{3,4}$ by the map $\psi_{34} \circ(0,8,4, v, 1,5, u)(2,6,7,3,9)$, where $\psi_{34}$ is as in Subcase 5.1 .

Subcase 6.6.2.2. $\operatorname{lk}(3)=C_{6}(8,4,0,2, u, 9)$. Completing successively, we get $1 \mathrm{k}(9)=$ $C_{6}(1,7,5, u, 3,8), \operatorname{lk}(8)=C_{6}(1,6, v, 4,3,9), \operatorname{lk}(6)=C_{6}(0,1,8, v, u, 5)$. Now, $M \cong$ $T_{6,2,2}$ by the map $\psi \circ(0,1, u, 7,4,3,8)(2,6, v, 5)$, where $\psi: V \rightarrow V\left(T_{6,2,2}\right)$ is given by $\psi(i)=u_{i}$, for $1 \leq i \leq 6, \psi(5+i)=v_{i}$, for $2 \leq i \leq 4, \psi(0)=v_{5}, \psi(u)=v_{6}$ and $\psi(v)=v_{7}$.

Lemma 3.11. If $M$ is a 14-vertex degree-regular combinatorial 2-manifolds of Euler characteristic 0 then $M$ is isomorphic to $T_{14,1,2}, T_{14,1,3}$ or $Q_{7,2}$.

Proof. Let $M$ be a 14-vertex degree regular combinatorial 2-manifold of Euler characteristic 0 . Let the vertex set $V$ be $\{0,1, \ldots, 9, u, v, w, z\}$. Let $\varphi: V \rightarrow\{1, \ldots, 14\}$ be given by $\varphi(i)=i$, for $1 \leq i \leq 9, \varphi(0)=10, \varphi(u)=11, \varphi(v)=12, \varphi(w)=13$ and $\varphi(z)=14$.

Since $\chi(M)=0$, the degree of each vertex is 6 . Assume without loss that $1 \mathrm{k}(0)=$ $C_{6}(1,2,3,4,5,6)$. By Lemma $3.8, \operatorname{lk}(1)=C_{6}(6,0,2,7, x, y)$, for some $x, y \in V$. It is easy to see that $(x, y)=(3,4),(3,8),(4,3),(4,8),(5,3),(5,4),(5,8),(8,3),(8,4)$, $(8,9)$. The case $(x, y)=(3,8)$ is isomorphic to the case $(x, y)=(5,8)$ by the map $(2,6)(3,5)(7,8)$ and to the case $(x, y)=(8,4)$ by the map $(0,1)(2,6)(3,4,8)(5,7)$. Hence we may assume that $(x, y)=(3,4),(3,8),(4,3),(4,8),(5,3),(5,4),(8,3),(8,9)$.

Claim. $(x, y)=(3,4),(8,3)$ or $(8,9)$

If $(x, y)=(4,3)$ then, considering the links of $3,6,2,4,7,8, u$ successively we get $C_{3}(v, u, 7) \subseteq \operatorname{lk}(w)$. So, $(x, y) \neq(4,3)$. If $(x, y)=(5,3)$ then, $\operatorname{lk}(5)=$ $C_{6}(1,3,6,0,4,7)$. But then $C_{4}(0,1,3,5) \subseteq \operatorname{lk}(6)$. So, $(x, y) \neq(5,3)$. Similarly, $(x, y) \neq(3,8),(4,8)$ or $(5,4)$. This proves the claim.

Case $1 .(x, y)=(3,4)$, i.e., $\operatorname{lk}(1)=C_{6}(6,0,2,7,3,4)$. Now, it easy to see that $\operatorname{lk}(3)=$ $C_{6}(7,1,4,0,2,5)$ or $C_{6}(7,1,4,0,2,8)$. In the first case, $\operatorname{lk}(5)=C_{6}(4,0,6,2,3,7)$ or $C_{6}(4,0,6,7,3,2)$. In both these cases we have 23 edges in $M[\{0, \ldots 7\}]$. This is not possible by Lemma 3.9. Thus, $\operatorname{lk}(3)=C_{6}(7,1,4,0,2,8)$. Now, $\operatorname{lk}(2)=C_{6}(7,1,0,3,8, b)$ for some $b \in V$. It is easy to see that $b=5$ or 9 . If $b=5$ then, considering the links of 4 and 5 , we get $\geq 17$ faces not containing any of $9, u, v, w, z$. This is not possible by Lemma 3.7. Thus $\operatorname{lk}(2)=C_{6}(7,1,0,3,8,9)$. Again, by using Lemma 3.7, we successively get $\operatorname{lk}(7)=C_{6}(8,3,1,2,9, u), \operatorname{lk}(8)=C_{6}(9,2,3,7, u, v), \operatorname{lk}(9)=C_{6}(u, 7,2,8, v, w)$, $\operatorname{lk}(u)=C_{6}(v, 8,7,9, w, z), \operatorname{lk}(v)=C_{6}(w, 9,8, u, z, 5)$. Then 045, 056, 5vw and 5vz are faces. So, $\operatorname{lk}(5)=C_{6}(6,0,4, w, v, z)$ or $C_{6}(6,0,4, z, v, w)$. In the first case, considering the links of 4 and 6 , we get $C_{5}(w, 6,5, v, u) \subseteq \operatorname{lk}(z)$. Thus $\operatorname{lk}(5)=C_{6}(6,0,4, z, v, w)$. 
Now, completing successively, we get $\operatorname{lk}(w)=C_{6}(9, v, 5,6, z, u)$ and $\operatorname{lk}(6)=$ $C_{6}(1,0,5, w, z, 4)$. Here $M$ is isomorphic to $T_{14,1,2}$ by the map $\varphi \circ(0,7,3,5)(1,6,9)(2$, $4,8)(u, z)(v, w)$.

Case 2. $(x, y)=(8,3)$. Since $023,034,136$ and 138 are faces, $\operatorname{lk}(3)=C_{6}(2,0,4,8,1,6)$ or $C_{6}(2,0,4,6,1,8)$. In the first case, considering the links of $6,2,9,7,8$ and 4 successively, we get 7 vertices in $\operatorname{lk}(u)$. Thus, $\operatorname{lk}(3)=C_{6}(2,0,4,6,1,8)$.

Now, completing successively, we get $\operatorname{lk}(6)=C_{6}(5,0,1,3,4,9), \operatorname{lk}(4)=C_{6}(5,0,3,6$, $9, u), \operatorname{lk}(5)=C_{6}(u, 4,0,6,9, v), \operatorname{lk}(9)=C_{6}(u, 4,6,5, v, w), \operatorname{lk}(2)=C_{6}(8,3,0,1$, $7, z), \operatorname{lk}(8)=C_{6}(7,1,3,2, z, w), \operatorname{lk}(u)=C_{6}(4,5, v, z, w, 9), \operatorname{lk}(z)=C_{6}(2,7, v, u$, $w, 8)$ and $\operatorname{lk}(7)=C_{6}(1,2, z, v, w, 8)$. Here $M \cong T_{14,1,2}$ by the map $\varphi \circ(0,6,7,1,4,8,2$, $3,5,9)$.

Case 3. $(x, y)=(8,9)$, i.e., $\operatorname{lk}(1)=C_{6}(6,0,2,7,8,9)$. Now, $\operatorname{lk}(6)=C_{6}(9,1,0,5$, $a, b)$, for some $a, b \in V$. It is easy to see that $(a, b)=(2,3),(2,7),(3,2),(3,4),(3,7)$, $(3, u),(7,2),(7,3),(7,4),(7, u),(8,3),(8,4),(8,7),(8, u),(u, 3),(u, 4),(u, 7),(u, v)$. Since the set of known faces is invariant under the map $(0,1)(3,7)(4,8)(5,9)$, we may assume that $(a, b)=(2,3),(2,7),(3,4),(3,7),(3, u),(7,3),(7,4),(7, u),(8,4),(8, u)$ or $(u, v)$.

By the similar arguments as in the previous claim one gets $(a, b)=(2,7)$ or $(u, v)$.

Subcase 3.1. $1 \mathrm{k}(6)=C_{6}(9,1,0,5,2,7)$. Completing successively, we get $1 \mathrm{k}(2)$ $=C_{6}(1,0,3,5,6,7), \operatorname{lk}(5)=C_{6}(4,0,6,2,3, u), \operatorname{lk}(3)=C_{6}(2,0,4, v, u, 5)$, $\operatorname{lk}(4)=C_{6}(3,0,5, u, w, v), \operatorname{lk}(u)=C_{6}(3,5,4, w, z, v), \operatorname{lk}(7)=C_{6}(1,2,6,9, z, 8)$, $\operatorname{lk}(9)=C_{6}(1,6,7, z, w, 8), \operatorname{lk}(z)=C_{6}(7,8, v, u, w, 9)$ and $\operatorname{lk}(v)=C_{6}(3,4, w, 8, z, u)$. Here $M$ is isomorphic to $T_{14,1,2}$ by the map $\varphi \circ(0,4,1,7,8)(2,5,3)(u, z)(v, w)$.

Subcase 3.2. $\operatorname{lk}(6)=C_{6}(9,1,0,5, u, v)$. Now, $\operatorname{lk}(5)=C_{6}(u, 6,0,4, c, d)$, for some $c, d \in V$. It is easy to see that $(c, d)=(2,3),(2,7),(7,2),(7,3),(7,8),(7, w),(8,3)$, $(8,7),(8,9),(8, w),(9,8),(v, 3),(v, 7),(v, 8),(v, 9),(v, w),(w, 3),(w, 7),(w, 8)$, $(w, z)$. Since the set of known faces is invariant under the map $(0,6)(2,9)(3, v)(4, u)(7,8)$, we may assume that $(c, d)=(2,3),(2,7),(7,2),(7,3),(7,8),(7, w),(8,3),(8,7)$, $(8, w),(v, 3),(v, w)$ or $(w, z)$.

Claim. $(c, d)=(7,8),(8, w)$ or $(w, z)$.

If $(c, d)=(2,3)$ then, $\operatorname{lk}(5)=C_{6}(u, 6,0,4,2,3)$. Considering the links of 2, 4, 3, $u, w$ successively, we get $C_{5}(8, w, 4,2,1) \subseteq \operatorname{lk}(7)$. If $(c, d)=(2,7)$ then, considering $\operatorname{lk}(2)$ we get $C_{4}(3,2,5,0) \subseteq 1 \mathrm{k}(4)$. If $(c, d)=(7,3)$ then, considering $\operatorname{lk}(3)$, we get $C_{4}(7,3,0,1) \subseteq 1 \mathrm{k}(2)$. If $(c, d)=(v, 3)$ then, considering $\mathrm{lk}(3)$, we get 7 vertices in $\operatorname{lk}(v)$. So, $(c, d) \neq(2,3),(2,7),(7,3)$ or $(v, 3)$. Similarly, $(c, d) \neq(7,2),(7, w),(8,3)$, $(8,7)$ or $(v, w)$. This proves the claim.

Subcase 3.2.1. $\operatorname{lk}(5)=C_{6}(u, 6,0,4,7,8)$. Now, $\operatorname{lk}(8)=C_{6}(u, 5,7,1,9, x)$, for some $x \in V$. It is easy to check that $x=3, w$. By using Lemma 3.7, we get $x \neq 3$. So, $\operatorname{lk}(8)=C_{6}(u, 5,7,1,9, w)$. This implies that $\operatorname{lk}(9)=C_{6}(w, 8,1,6, v, y)$, for some $y \in V$. It is easy to see that $y=3$ or $z$.

Subcase 3.2.1.1. $1 \mathrm{k}(9)=C_{6}(w, 8,1,6, v, 3)$. This implies that $1 \mathrm{k}(u)=C_{6}(w, 8,5,6$, $v, z)$. Again, by using Lemma 3.7, we get $\operatorname{lk}(7)=C_{6}(4,5,8,1,2, z)$. Then $1 \mathrm{k}(4)=$ $C_{6}(3,0,5,7, z, a)$, for some $a \in V$. Considering lk(3), we get $a=v$ or $w$. 
Subcase 3.2.1.1.1. $\operatorname{lk}(4)=C_{6}(3,0,5,7, z, v)$. Completing successively we get $\operatorname{lk}(z)=C_{6}(2,7,4, v, u, w), \operatorname{lk}(2)=C_{6}(1,0,3, w, z, 7)$ and $\operatorname{lk}(3)=C_{6}(2,0,4, v, 9, w)$. Here $M \cong T_{14,1,3}$ by the map $\varphi \circ(0,1,4, v, w, 6, z, 9,3,2,5, u)(7,8)$.

Subcase 3.2.1.1.2. $1 \mathrm{k}(4)=C_{6}(3,0,5,7, z, w)$. Completing successively, we get $\operatorname{lk}(3)=C_{6}(2,0,4, w, 9, v), \operatorname{lk}(2)=C_{6}(1,0,3, v, z, 7)$ and $\operatorname{lk}(v)=C_{6}(2,3,9,6, u, z)$. Now, $M \cong Q_{7,2}$ by the map $\varphi \circ(0,1,9, u, v, 5,2,7,8)(3, w, 4, z, 6)$.

Subcase 3.2.1.2. $\operatorname{lk}(9)=C_{6}(w, 8,1,6, v, z)$. Then it follows that $\operatorname{lk}(u)=C_{6}(v, 6,5$, $8, w, 3)$. This case is now isomorphic to the Subcase 3.2.1.1 by the map $(1,5)(2,4)(u, 9)$.

Subcase 3.2.2. $\operatorname{lk}(5)=C_{6}(u, 6,0,4,8, w)$. Since, $178,189,458$ and $58 w$ are faces, $\operatorname{lk}(8)=C_{6}(4,5, w, 9,1,7)$ or $C_{6}(4,5, w, 7,1,9)$.

Subcase 3.2.2.1. $\operatorname{lk}(8)=C_{6}(4,5, w, 9,1,7)$. Then $\operatorname{lk}(4)=C_{6}(3,0,5,8,7, x)$, for some $x \in V$. It is easy to see that $x=v, z$. If $x=v$ then, considering the links of $4,7, v$ successively, we obtain 29 faces in $M$, which is not possible. Thus $1 \mathrm{k}(4)=C_{6}(3,0,5,8,7, z)$.

Completing successively, we get $\operatorname{lk}(7)=C_{6}(2,1,8,4, z, v), \operatorname{lk}(2)=C_{6}(3,0,1,7$, $v, u), \operatorname{lk}(v)=C_{6}(2, u, 6,9, z, 7), \operatorname{lk}(9)=C_{6}(1,6, v, z, w, 8), \operatorname{lk}(z)=C_{6}(3,4,7, v$, $9, w)$ and $\operatorname{lk}(w)=C_{6}(3, u, 5,8,9, z)$. Here $M \cong T_{14,1,3}$ by the map $\varphi \circ(0,6,2,9,1,5,3)$ $(4,7,8)(u, w, z)$.

Subcase 3.2.2.2. $\operatorname{lk}(8)=C_{6}(4,5, w, 7,1,9)$. Then $\operatorname{lk}(4)=C_{6}(3,0,5,8,9, z)$ and $\operatorname{lk}(9)=C_{6}(1,6, v, z, 4,8)$. Now, $\operatorname{lk}(w)=C_{6}(u, 5,8,7, a, b)$, for some $a, b \in V$. It is easy to check that $(a, b)=(3,2),(3, z),(v, z)$ or $(z, 3)$. The set of known faces is invariant under the map $(0,9)(1,6)(2, v)(3, z)(5,8)(7, u)$. So, we may assume that $(a, b)=(3,2)$, $(3, z)$ or $(z, 3)$. If $(a, b)=(3,2)$ then, considering the links of 2 and 3 , we get 7 vertices in $1 \mathrm{k}(7)$. If $(a, b)=(3, z)$ then, considering $1 \mathrm{k}(3)$ we get $C_{4}(7,3,0,1) \subseteq 1 \mathrm{k}(2)$. So, $\operatorname{lk}(w)=C_{6}(u, 5,8,7, z, 3)$.

Completing successively, we get $\operatorname{lk}(3)=C_{6}(2,0,4, z, w, u), \operatorname{lk}(u)=C_{6}(2,3, w, 5$, $6, v), \operatorname{lk}(2)=C_{6}(1,0,3, u, v, 7)$ and $\operatorname{lk}(z)=C_{6}(3,4,9, v, 7, w)$. Here $M \cong Q_{7,2}$ by the $\operatorname{map} \varphi \circ(0,1, w)(2, z, 4,3)(5,9)(6,7, v)(8, u)$.

Subcase 3.2.3. $\operatorname{lk}(5)=C_{6}(u, 6,0,4, w, z)$. This implies that $\operatorname{lk}(4)=C_{6}(w, 5,0,3, x, y)$, for some $x, y \in V$. It is easy to check that $(x, y)=(7,2),(7,8),(7, v),(8,7),(8,9)$, $(9, v),(u, v),(v, 7),(v, 8),(v, 9),(v, u),(z, 7),(z, 8),(z, u)$ or $(z, v)$. By similar arguments as in the previous claims one gets $(x, y)=(8,7),(8,9)$ or $(9, v)$.

Subcase 3.2.3.1. $\mathrm{lk}(4)=C_{6}(w, 5,0,3,8,9)$. Completing successively, we get $1 \mathrm{k}(9)$ $=C_{6}(w, 4,8,1,6, v), \operatorname{lk}(w)=C_{6}(v, 9,4,5, z, 7), \operatorname{lk}(8)=C_{6}(3,4,9,1,7, z), \operatorname{lk}(7)=$ $C_{6}(2,1,8, z, w, v), \operatorname{lk}(z)=C_{6}(3,8,7, w, 5, u), \operatorname{lk}(v)=C_{6}(2,7, w, 9,6, u)$ and $\operatorname{lk}(u)$ $=C_{6}(2,3, z, 5,6, v)$. Here $M \cong T_{14,1,3}$ by the map $\varphi \circ(0,5,1,6,2,9,3,8,7)(u, v, w, z)$.

Subcase 3.2.3.2. $1 \mathrm{k}(4)=C_{6}(w, 5,0,3,9, v)$. Completing successively, we get $1 \mathrm{k}(9)$ $=C_{6}(3,4, v, 6,1,8), \operatorname{lk}(3)=C_{6}(2,0,4,9,8, z), \operatorname{lk}(v)=C_{6}(u, 6,9,4, w, 7), \operatorname{lk}(2)=$ $C_{6}(1,0,3, z, u, 7), \operatorname{lk}(8)=C_{7}(1,9,3, z, w, 7), \operatorname{lk}(z)=C_{6}(2,3,8, w, 5, u), \operatorname{lk}(w)$ $=C_{6}(4,5, z, 8,7, v)$ and $\operatorname{lk}(7)=C_{6}(1,2, u, v, w, 8)$. Here $M \cong T_{14,1,3}$ by the map $\varphi \circ(0,1,5, z, w)(3, v, 7,6,4, u)(8,9)$.

Subcase 3.2.3.3. $\operatorname{lk}(4)=C_{6}(w, 5,0,3,8,7)$. Completing successively we get $1 \mathrm{k}(7)$ $=C_{6}(w, 4,8,1,2, v), \operatorname{lk}(8)=C_{6}(3,4,7,1,9, z), \operatorname{lk}(9)=C_{6}(z, 8,1,6, v, w), \operatorname{lk}(v)=$ 
$C_{6}(2,7, w, 9,6, u), \operatorname{lk}(z)=C_{6}(5, w, 9,8,3, u), \operatorname{lk}(u)=C_{6}(2, v, 6,5, z, 3)$ and $\operatorname{lk}(2)=$ $C_{6}(1,0,3, u, v, 7)$. Here $M \cong Q_{7,2}$ by the map given by $\varphi \circ(0,1,3,7, u, 8,5, z, 6,2,9,4$, $w, v)$.

Lemma 3.12. If $M$ is a 15-vertex degree-regular combinatorial 2-manifolds of Euler characteristic 0 then $M$ is isomorphic to $T_{15,1,2}, \ldots, T_{15,1,5}, B_{3,5}, B_{5,3}$ or $Q_{5,3}$.

Proof. Let $M$ be a 15-vertex degree regular combinatorial 2-manifold of Euler characteristic 0 . Let the vertex set $V$ be $\{0,1, \ldots, 9, u, v, w, z, s\}$. Let $\varphi: V \rightarrow\{1, \ldots, 15\}$ be given by $\varphi(i)=i$, for $1 \leq i \leq 9, \varphi(0)=10, \varphi(u)=11, \varphi(v)=12, \varphi(w)=13, \varphi(z)=14$ and $\varphi(s)=15$.

Since $\chi(M)=0$, the degree of each vertex is 6 . As earlier, we may assume that $\mathrm{lk}(0)=$ $C_{6}(1,2,3,4,5,6)$. By Lemma 3.8, $\operatorname{lk}(1)=C_{6}(7,2,0,6, x, y)$, for some $x, y \in V$. It is easy to see that $(x, y)=(3,4),(3,5),(3,8),(4,3),(4,5),(4,8),(8,3),(8,4),(8,5)$, $(8,9)$.

If $(x, y)=(3,5)$ then, considering $\operatorname{lk}(3)$ we get 7 vertices in $\operatorname{lk}(5)$. The case $(x, y)=$ $(8,3)$ is isomorphic to the case $(x, y)=(4,8)$ by the map $(0,1)(2,6)(3,4,8)(5,7)$ and the case $(x, y)=(8,5)$ is isomorphic to the case $(x, y)=(8,3)$ by the map $(2,6)(3,5)(7,8)$. So, we may assume that $(x, y)=(3,4),(3,8),(4,3),(4,5),(4,8),(8,4)$ or $(8,9)$.

Case 1. $(x, y)=(3,4)$, i.e., $\operatorname{lk}(1)=C_{6}(7,2,0,6,3,4)$. Then $\operatorname{lk}(3)=C_{6}(2,0,4,1$, $6,8), \operatorname{lk}(6)=C_{6}(5,0,1,3,8,9), \operatorname{lk}(4)=C_{6}(5,0,3,1,7, u)$ and $\operatorname{lk}(2)=C_{6}(8,3,0,1$, $7, v)$. Now, it is easy to see that $\operatorname{lk}(8)=C_{6}(9,6,3,2, v, u)$ or $C_{6}(9,6,3,2, v, w)$. In the first case, we get 34 edges in $M[\{0, \ldots, 9, u, v\}]$, a contradiction to Lemma 3.9. So, $\operatorname{lk}(8)$ $=C_{6}(9,6,3,2, v, w)$. Now, completing successively, we get $\operatorname{lk}(7)=C_{6}(u, 4,1,2, v, z)$, $\operatorname{lk}(v)=C_{6}(w, 8,2,7, z, s), \operatorname{lk}(5)=C_{6}(4,0,6,9, s, u), \operatorname{lk}(s)=C_{6}(5,9, z, v, w, u)$, $\operatorname{lk}(u)=C_{6}(7,4,5, s, w, z)$ and $\operatorname{lk}(9)=C_{6}(5,6,8, w, z, s)$. Here $M \cong B_{3,5}$ by the map $\psi_{35} \circ(2,9, z)(0, v, 6, u, 1,8)(3,7,5, s)$, where $\psi_{35}: V \rightarrow V\left(B_{3,5}\right)$ given by $\psi_{35}(i)=$ $v_{1 i}, \psi_{35}(3+i)=v_{2 i}, \psi_{35}(6+i)=v_{3 i}, 1 \leq i \leq 3, \psi_{35}(0)=v_{41}, \psi_{35}(u)=v_{42}$, $\psi_{35}(v)=v_{43}, \psi_{35}(w)=v_{51}, \psi_{35}(z)=v_{52}$ and $\psi_{35}(s)=v_{53}$.

Case 2. $(x, y)=(3,8)$. Then $\operatorname{lk}(3)=C_{6}(2,0,4,8,1,6)$ or $C_{6}(2,0,4,6,1,8)$.

Subcase 2.1. $1 \mathrm{k}(3)=C_{6}(2,0,4,8,1,6)$. Completing successively, we get $1 \mathrm{k}(2)=C_{6}(7$, $1,0,3,6,9), \operatorname{lk}(6)=C_{6}(5,0,1,3,2,9), \operatorname{lk}(9)=C_{6}(5,6,2,7, v, u), \operatorname{lk}(5)=C_{6}(4,0$, $6,9, u, w), \operatorname{lk}(7)=C_{6}(8,1,2,9, v, z), \operatorname{lk}(8)=C_{6}(4,3,1,7, z, s), \operatorname{lk}(4)=C_{6}(5,0,3,8$, $s, w), \operatorname{lk}(u)=C_{6}(5,9, v, s, z, w), \operatorname{lk}(s)=C_{6}(4,8, z, u, v, w)$ and $\operatorname{lk}(z)=C_{6}(7,8, s, u$, $w, v)$. Here $M$ is isomorphic to $Q_{5,3}$ by the map $\psi \circ(5,8, s, v, 9,7,6)(0,1,3,2,4, z, w, u)$, where $\psi: V \rightarrow V\left(Q_{5,3}\right)$ is given by $\psi(i)=u_{i 1}, \psi(5+i)=u_{i 2}, 1 \leq i \leq 3$, $\psi(3+j)=v_{j 1}, 1 \leq j \leq 2, \psi(9)=v_{12}, \psi(0)=v_{22}, \psi(u)=u_{13}, \psi(v)=u_{23}$, $\psi(w)=u_{33}, \psi(z)=v_{13}$ and $\psi(s)=v_{23}$.

Subcase 2.2. $\operatorname{lk}(3)=C_{6}(2,0,4,6,1,8)$. Completing successively, we get $1 \mathrm{k}(2)$ $=C_{6}(7,1,0,3,8,9), \operatorname{lk}(8)=C_{6}(7,1,3,2,9, u), \operatorname{lk}(6)=C_{6}(5,0,1,3,4, v)$, $\operatorname{lk}(4)=C_{6}(5,0,3,6, v, w), \operatorname{lk}(7)=C_{6}(9,2,1,8, u, z), \operatorname{lk}(5)=C_{6}(v, 6,0,4, w, s)$, $\operatorname{lk}(9)=C_{6}(8,2,7, z, s, u), \operatorname{lk}(v)=C_{6}(6,4, w, z, s, 5), \operatorname{lk}(s)=C_{6}(u, w, 5, v, z, 9)$ and $\operatorname{lk}(u)=C_{6}(7,8,9, s, w, z)$. Here $M \cong T_{15,1,2}$ by the map $\varphi \circ(0,7,2,4,9,1$, 5) $(3,6,8)(u, s, w, v)$.

Case 3. $(x, y)=(4,3)$, i.e., $\operatorname{lk}(1)=C_{6}(7,2,0,6,4,3)$. Then $\operatorname{lk}(4)=C_{6}(5,0,3,1$, $6,8), \operatorname{lk}(3)=C_{6}(2,0,4,1,7,9), \operatorname{lk}(6)=C_{6}(5,0,1,4,8, u), \operatorname{lk}(2)=C_{6}(9,3,0,1,7, v)$, 
$\operatorname{lk}(7)=C_{6}(9,3,1,2, v, w), \operatorname{lk}(5)=C_{6}(8,4,0,6, u, z), \operatorname{lk}(8)=C_{6}(u, 6,4,5, z, s)$, $\operatorname{lk}(9)=C_{6}(v, 2,3,7, w, s)$. Thus, $\operatorname{lk}(s)=C_{6}(v, 9, w, z, 8, u)$ or $C_{6}(v, 9, w, u, 8, z)$. In the first case, considering the links of $s, u, v$ successively, we get 7 vertices in $\operatorname{lk}(v)$. So, $\operatorname{lk}(s)=C_{6}(v, 9, w, u, 8, z)$. Now, completing successively, we get $\operatorname{lk}(u)=C_{6}(5,6,8, s, w, z)$ and $\operatorname{lk}(z)=C_{6}(5,8, s, v, w, u)$. Here $M \cong T_{15,1,2}$ by the $\operatorname{map} \varphi \circ(0,5,2,8,1,6,3,7,9)(u, s, w, v)$.

Case 4. $(x, y)=(4,5)$, i.e., $\operatorname{lk}(1)=C_{6}(7,2,0,6,4,5)$. Now, completing successively, we get $\operatorname{lk}(4)=C_{6}(3,0,5,1,6,8), \operatorname{lk}(6)=C_{6}(5,0,1,4,8,9), 1 \mathrm{k}(5)$ $=C_{6}(7,1,4,0,6,9), \operatorname{lk}(7)=C_{6}(2,1,5,9, v, u), \operatorname{lk}(2)=C_{6}(3,0,1,7, u, w)$, $\operatorname{lk}(3)=C_{6}(8,4,0,2, w, z), \operatorname{lk}(8)=C_{6}(9,6,4,3, z, s), \operatorname{lk}(9)=C_{6}(7,5,6,8, s, v)$, $\operatorname{lk}(v)=C_{6}(u, 7,9, s, w, z), \operatorname{lk}(z)=C_{6}(w, 3,8, s, u, v)$ and $\operatorname{lk}(u)=C_{6}(2,7, v, z, s, w)$. Now, $M \cong Q_{5,3}$ by the map $\psi \circ(0,1,2, z)(3,8,7, s, 9,6,4,5)(u, v, w)$, where $\psi$ is as in Subcase 2.1.

Case 5. $(x, y)=(4,8)$, i.e., $\operatorname{lk}(1)=C_{6}(7,2,0,6,4,8)$. Now, completing successively, we get $\operatorname{lk}(4)=C_{6}(5,0,3,6,1,8), \operatorname{lk}(6)=C_{6}(5,0,1,4,3,9), 1 \mathrm{k}(3)$ $=C_{6}(2,0,4,6,9, u), \operatorname{lk}(2)=C_{6}(7,1,0,3, u, v), \operatorname{lk}(5)=C_{6}(8,4,0,6,9, w)$, $\operatorname{lk}(8)=C_{6}(7,1,4,5, w, z), \operatorname{lk}(9)=C_{6}(u, 3,6,5, w, s), \operatorname{lk}(7)=C_{6}(v, 2,1,8, z, s)$, $\operatorname{lk}(s)=C_{6}(w, 9, u, z, 7, v), \operatorname{lk}(w)=C_{6}(8,5,9, s, v, z)$ and $\operatorname{lk}(z)=C_{6}(7,8, w, v, u, s)$. Now, $M$ is isomorphic to $B_{3,5}$ by the map $\psi_{35} \circ(1, u)(2, s)(5,9)(0, v, 3,4,7, z, w, 6,8)$, where $\psi_{35}$ is as in Case 1.

Case 6. $(x, y)=(8,4)$, i.e., $\operatorname{lk}(1)=C_{6}(7,2,0,6,8,4)$. Now, $\operatorname{lk}(4)=C_{6}(5,0,3,8,1,7)$ or $C_{6}(5,0,3,7,1,8)$. In the first case, we get $\operatorname{lk}(3)=C_{6}(8,4,0,2, a, b)$, for some $a, b \in V$. Using Lemma 3.9, we may assume that $(a, b)=(9, u)$. Considering the links of $2,7,5,6,8,9, s, u$ and $v$ successively, we get 7 vertices in $\operatorname{lk}(v)$. Thus, $\operatorname{lk}(4)=C_{6}(5,0,3,7,1,8)$.

Again, by using Lemma 3.9, we get $\operatorname{lk}(3)=C_{6}(7,4,0,2,9, u), \operatorname{lk}(2)=C_{6}(7,1,0,3$, $9, v), \operatorname{lk}(7)=C_{6}(2,1,4,3, u, v), \operatorname{lk}(9)=C_{6}(2,3, u, z, w, v), \operatorname{lk}(v)=C_{6}(u, 7,2,9$, $w, s), \operatorname{lk}(u)=C_{6}(9,3,7, v, s, z)$. Then $\operatorname{lk}(z)=C_{6}(s, u, 9, w, a, b)$, for some $a, b \in V$. It is easy to see that $(a, b)=(5,6),(5,8),(6,5),(6,8),(8,5)$ or $(8,6)$. Since the set of known faces remain invariant under the map $(0,4)(2,7)(6,8)(9, u)(w, s)$, we can assume that $(a, b)=(5,6),(5,8),(6,8)$ or $(8,6)$.

Subcase 6.1. $(a, b)=(5,6)$. Completing successively, we get $\operatorname{lk}(5)=C_{6}(8,4,0,6$, $z, w), \operatorname{lk}(6)=C_{6}(8,1,0,5, z, s)$ and $\operatorname{lk}(8)=C_{6}(5,4,1,6, s, w)$. Now, $M$ is isomorphic to $B_{5,3}$ by the map $\psi_{53} \circ(0, s, 3,1)(2,6,9,7, u)(4,5, z, 8)$, where $\psi_{53}: V \rightarrow V\left(B_{5,3}\right)$ is given by $\psi_{53}(i)=v_{1 i}$, for $1 \leq i \leq 5, \psi_{53}(5+i)=v_{2 i}$, for $1 \leq i \leq 4, \psi_{53}(0)=v_{25}$, $\psi_{53}(u)=v_{31}, \psi_{53}(v)=v_{32}, \psi_{53}(w)=v_{33}, \psi_{53}(z)=v_{34}, \psi_{53}(s)=v_{35}$.

Subcase 6.2. $(a, b)=(5,8)$. Completing successively, we get $\operatorname{lk}(5)=C_{6}(w, z, 8,4$, $0,6), \operatorname{lk}(8)=C_{6}(6,1,4,5, z, s)$ and $\operatorname{lk}(6)=C_{6}(5,0,1,8, s, w)$. Here $M$ is isomorphic to $T_{15,1,5}$ by the map $\varphi \circ(0,2,1,7,6,8, w, 9)(3, u, 5)(4, v, s, z)$.

Subcase 6.3. $(a, b)=(6,8)$. Completing successively, we get $\operatorname{lk}(6)=C_{6}(5,0,1,8, z$, $w), \operatorname{lk}(8)=C_{6}(1,4,5, s, z, 6)$ and $\left.\operatorname{lk}(5)=4,0,6, w, s, 8\right)$. Here $M \cong B_{5,3}$ by the map $\psi_{53} \circ(1,9)(0, z, 2, s, v, 6,8,3,5, w, 7)$, where $\psi_{53}$ is as in Subcase 6.1 .

Subcase 6.4. $(a, b)=(8,6)$. Completing successively, we get lk $(6)=C_{6}(5,0,1,8, z, s)$, $\operatorname{lk}(8)=C_{6}(5,4,1,6, z, w)$ and $\operatorname{lk}(5)=C_{6}(8,4,0,6, s, w)$. Here $M$ is isomorphic to $T_{15,1,4}$ by the map given by $\varphi \circ(0,7,1,2,6,3, u, s, 4, v, 5,8, w, 9)$. 
Case 7. $(x, y)=(8,9)$, i.e., $\operatorname{lk}(1)=C_{6}(7,2,0,6,8,9)$. Now, $\operatorname{lk}(6)=C_{6}(8,1,0,5$, $a, b)$. It is easy to check that $(a, b)=(2,3),(2,7),(3,2),(3,7),(3,4),(3, u),(7,2)$, $(7,3),(7,4),(7, u),(9,3),(9,4),(9,7),(9, u),(u, 3),(u, 4),(u, 7),(u, v)$.

If $(a, b)=(3,7)$ then, considering links of 3 and 7 , we get 7 vertices in $1 \mathrm{k}(7)$. If $(a, b)=(7,3),(7,4)$ or $(9,4)$ then, considering $\operatorname{lk}(b)$, we get 7 vertices in $\operatorname{lk}(a)$. Since the set of known faces remain invariant under that map $(0,1)(3,7)(4,9)(5,8)$, we may assume that $(a, b)=(2,3),(2,7),(3,4),(3, u),(7, u),(9, u),(u, v)$.

Subcase 7.1. $\operatorname{lk}(6)=C_{6}(8,1,0,5,2,3)$. Completing successively, we get $1 \mathrm{k}(2)=$ $C_{6}(1,0,3,6,5,7), \operatorname{lk}(5)=C_{6}(4,0,6,2,7, u), \operatorname{lk}(3)=C_{6}(4,0,2,6,8, v), 1 \mathrm{k}(8)$ $=C_{6}(9,1,6,3, v, w), \operatorname{lk}(4)=C_{6}(u, 5,0,3, v, z), \operatorname{lk}(v)=C_{6}(w, 8,3,4, z, s), \operatorname{lk}(7)=$ $C_{6}(9,1,2,5, u, s), 1 \mathrm{k}(9)=C_{6}(w, 8,1,7, s, z), \operatorname{lk}(z)=C_{6}(4, v, s, 9, w, u)$ and $\operatorname{lk}(s)=$ $C_{6}(w, u, 7,9, z, v)$. Here $M \cong B_{3,5}$ by the map $\psi_{35} \circ(0,7,5,9,1,4)(2,8, s)(3, u, 6, v$, $z, w)$, where $\psi_{35}$ is as in Case 1.

Subcase 7.2. $\operatorname{lk}(6)=C_{6}(8,1,0,5,2,7)$. Completing successively, we get $1 \mathrm{k}(2)$ $=C_{6}(5,6,7,1,0,3), \operatorname{lk}(5)=C_{6}(4,0,6,2,3, u), 1 \mathrm{k}(3)=C_{6}(4,0,2,5, u, v), 1 \mathrm{k}$ (4) $=C_{6}(u, 5,0,3, v, w), \operatorname{lk}(u)=C_{6}(v, 3,5,4, w, z), \operatorname{lk}(7)=C_{6}(9,1,2,6,8, s)$, $\operatorname{lk}(v)=C_{6}(w, 4,3, u, z, s), \operatorname{lk}(8)=C_{6}(9,1,6,7, s, z), \operatorname{lk}(9)=C_{6}(8,1,7, s, w, z)$ and $\operatorname{lk}(s)=C_{6}(8,7,9, w, v, z)$. Here $M \cong T_{15,1,2}$ by the map $\varphi \circ(0,7,3,9,1,4)(2,6,5,8)$.

Subcase 7.3. $1 \mathrm{k}(6)=C_{6}(8,1,0,5,3,4)$. Completing successively, we get $1 \mathrm{k}(3)$ $=C_{6}(2,0,4,6,5, u), 1 \mathrm{k}(4)=C_{6}(5,0,3,6,8, v), \operatorname{lk}(5)=C_{6}(3,6,0,4, v, u), 1 \mathrm{k}(2)$ $=C_{6}(7,1,0,3, u, w), \operatorname{lk}(u)=C_{6}(v, 5,3,2, w, z), \operatorname{lk}(v)=C_{6}(8,4,5, u, z, s), \operatorname{lk}(8)=$ $C_{6}(9,1,6,4, v, s), \operatorname{lk}(9)=C_{6}(7,1,8, s, w, z), \operatorname{lk}(w)=C_{6}(2,7, s, 9, z, u)$ and $\operatorname{lk}(7)=$ $C_{6}(9,1,2, w, s, z)$. Here $M \cong Q_{5,3}$ by the map $\psi \circ(0,1,8,7, u, s, 9)(2, z, w, v, 6,5,3)$, where $\psi$ is as in Subcase 2.1.

Subcase 7.4. $\operatorname{lk}(6)=C_{6}(8,1,0,5,3, u)$. Completing successively, we get $1 \mathrm{k}(3)$ $=C_{6}(4,0,2,5,6, u), \operatorname{lk}(2)=C_{6}(7,1,0,3,5, v), \operatorname{lk}(5)=C_{6}(4,0,6,3,2, v), 1 \mathrm{k}(4)$ $=C_{6}(u, 3,0,5, v, w), \operatorname{lk}(u)=C_{6}(8,6,3,4, w, z), \operatorname{lk}(8)=C_{6}(9,1,6, u, z, s), \operatorname{lk}(v)=$ $C_{6}(7,2,5,4, w, s), \operatorname{lk}(7)=C_{6}(9,1,2, v, s, z), \operatorname{lk}(s)=C_{6}(9, w, v, 7, z, 8)$ and $\operatorname{lk}(z)=$ $C_{6}(w, 9,7, s, 8, u)$. Here $M \cong B_{3,5}$ by the map $\psi_{35} \circ(0,7, z, 2, u, 5, v, s, 3,8,6,9, w, 1)$, where $\psi_{35}$ is as defined in Case 1 .

Subcase 7.5. $\operatorname{lk}(6)=C_{6}(8,1,0,5,7, u)$. Now, it is easy to see that $\operatorname{lk}(7)=C_{6}(9,1,2,5$, $6, u)$ or $C_{6}(9,1,2, u, 6,5)$. The first case is isomorphic to Subcase 6.2 by that map $(0,3,9,6,4, u, 8,5,7,1)$. The second case is isomorphic to Subcase 6.1 by the map $(0,3,9,6,4, u, 7,1)(5,8)$.

Subcase 7.6. $\operatorname{lk}(6)=C_{6}(8,1,0,5,9, u)$. Completing successively, we get $1 \mathrm{k}(9)$ $=C_{6}(8,1,7, u, 6,5), \operatorname{lk}(5)=C_{6}(4,0,6,9,8, v), \operatorname{lk}(8)=C_{6}(6,1,9,5, v, u), \operatorname{lk}(u)$ $=C_{6}(7,9,6,8, v, w), \operatorname{lk}(7)=C_{6}(2,1,9, u, w, z), \operatorname{lk}(v)=C_{6}(4,5,8, u, w, s), \operatorname{lk}(2)=$ $C_{6}(3,0,1,7, z, s), \operatorname{lk}(s)=C_{6}(3,2, z, 4, v, w), \operatorname{lk}(4)=C_{6}(3,0,5, v, s, z)$ and $\operatorname{lk}(3)=$ $C_{6}(4,0,2, s, w, z)$. Here $M \cong B_{3,5}$ by the map $\psi_{35} \circ(1, z, v, 5,6, w, 4,9,3,7, s, 8,2, u)$, where $\psi_{35}$ is as in Case 1.

Subcase 7.7. $\operatorname{lk}(6)=C_{6}(8,1,0,5, u, v)$. Then, $\operatorname{lk}(5)=C_{6}(u, 6,0,4, c, d)$, for some $c, d \in V$. It is easy to see that $(c, d)=(2,3),(2,7),(7,2),(7,3),(7,9)$, $(7, w),(8,9),(9,3),(9,7),(9,8),(9, w),(v, 3),(v, 7),(v, 8),(v, 9),(v, w),(w, 3)$, 
$(w, 7),(w, 9),(w, z)$. Since the set of known faces remain invariant under the map $(0,6)(2,8)(3, v)(4, u)(7,9)$, we may assume that $(c, d)=(2,3),(2,7),(7,2),(7,3)$, $(7,9),(7, w),(9,3),(9,7),(9, w),(v, 3),(v, w),(w, z)$.

Claim. $(c, d)=(2,3),(9, w),(v, w)$ or $(w, z)$.

If $(c, d)=(2,7)$ then, considering lk (2) we get $C_{4}(3,2,5,0) \subseteq \operatorname{lk}(4)$. If $(c, d)=(7,3)$ then, considering $\mathrm{lk}(3)$, we get $C_{4}(7,3,0,1) \subseteq \operatorname{lk}(2)$.

If $(c, d)=(7,2)$ then, considering the links of $2, u, 3,4,7, w, 9, v$ successively, we get 7 vertices in $1 \mathrm{k}(8)$. If $(c, d)=(9,3)$ or $(v, 3)$ then, considering $\mathrm{lk}(3)$, we get 7 vertices in $\operatorname{lk}(c)$. If $(c, d)=(9,7)$ then, considering the links of $9,4,7,2, v, 8, s$ successively, we get 7 vertices in $\operatorname{lk}(u)$. If $(c, d)=(7,9)$ then, considering the links of 5, 7, 2, 4, 3,v successively, we get $\operatorname{lk}(u)=C_{6}(9,5,6, v, z, x)$, where $x=z$ or $s$. In either case $\operatorname{lk}(x)$ has $\geq 7$ vertices.

If $(c, d)=(7, w)$ then, $\operatorname{lk}(7)=C_{6}(2,1,9, w, 5,4)$ or $C_{6}(2,1,9,4,5, w)$. In the first case, considering the links of 4 and 2 we get $C_{4}(z, 2,0,4) \subseteq 1 \mathrm{k}(3)$. In the second case, considering links of $7,4,2, w, z, u$ successively, we get 7 vertices in $\operatorname{lk}(3)$. This proves the claim.

Subcase 7.7.1. $\operatorname{lk}(5)=C_{6}(u, 6,0,4,2,3)$. Now, completing successively we get $1 \mathrm{k}(2)$ $=C_{6}(1,0,3,5,4,7), \operatorname{lk}(4)=C_{6}(3,0,5,2,7, w), \operatorname{lk}(3)=C_{6}(5,2,0,4, w, u), \operatorname{lk}(u)=$ $C_{6}(6,5,3, w, s, v), \operatorname{lk}(7)=C_{6}(9,1,2,4, w, z), \operatorname{lk}(w)=C_{6}(3,4,7, z, s, u), 1 \mathrm{k}(8)=$ $C_{6}(9,1,6, v, z, s), \operatorname{lk}(9)=C_{6}(8,1,7, z, v, s)$ and $\operatorname{lk}(v)=C_{6}(8,6, u, s, 9, z)$. Here $M$ is isomorphic to $Q_{5,3}$ by the map $\psi \circ(0,1, z, w, 6,8, u, 7, s, 9, v)(3,4)$, where $\psi$ is as in Subcase 2.1.

Subcase 7.7.2. $\operatorname{lk}(5)=C_{6}(u, 6,0,4,9, w)$. This implies that $\operatorname{lk}(9)=C_{6}(7,1,8,4,5, w)$ or $C_{6}(7,1,8, w, 5,4)$. In the first case, considering links of $9,4,8, v$ successively we see that $\operatorname{lk}(v)$ can not be a 6-cycle. Thus $\operatorname{lk}(9)=C_{6}(7,1,8, w, 5,4)$.

Now, completing successively, we get $\operatorname{lk}(4)=C_{6}(3,0,5,9,7, z), \operatorname{lk}(7)=C_{6}(2,1,9$, $4, z, s), \operatorname{lk}(2)=C_{6}(3,0,1,7, s, v), \operatorname{lk}(3)=C_{6}(z, 4,0,2, v, u), \operatorname{lk}(u)=C_{6}(5,6, v, 3$, $z, w), \operatorname{lk}(v)=C_{6}(6, u, 3,2, s, 8), \operatorname{lk}(8)=C_{6}(9,1,6, v, s, w)$ and $\operatorname{lk}(z)=C_{6}(7,4,3, u$, $w, s)$. Here $M \cong T_{15,1,3}$ by the map $\varphi \circ(0,5,8)(1,6,9,7,3)(u, v, w)(z, s)$.

Subcase 7.7.3. $\operatorname{lk}(5)=C_{6}(u, 6,0,4, v, w)$. Now, completing successively we get $\operatorname{lk}(v)$ $=C_{6}(8,6, u, 4,5, w), \operatorname{lk}(4)=C_{6}(3,0,5, v, u, z), \operatorname{lk}(u)=C_{6}(5,6, v, 4, z, w), \operatorname{lk}(w)=$ $C_{6}(8, v, 5, u, z, s), \operatorname{lk}(8)=C_{6}(9,1,6, v, w, s), \operatorname{lk}(z)=C_{6}(3,4, u, w, s, 7), \operatorname{lk}(7)=$ $C_{6}(2,1,9,3, z, s), \operatorname{lk}(3)=C_{6}(2,0,4, z, 7,9)$ and $\operatorname{lk}(2)=C_{6}(7,1,0,3,9, s)$. Here $M$ is isomorphic to $B_{3,5}$ by the map $\psi_{35} \circ(1,9,8,5, w)(2,7, v)(3, u)(4, z, s)$, where $\psi_{35}$ is as in Case 1.

Subcase 7.7.4. $\operatorname{lk}(5)=C_{6}(u, 6,0,4, w, z)$. Then $\operatorname{lk}(4)=C_{6}(w, 5,0,3, x, y)$, for some $x, y \in V$. It is easy to see that $(x, y)=(7,2),(7,9),(7, v),(7, s),(8,9),(8, v),(9,8)$, $(9, v),(9, s),(u, v),(v, 8),(v, 9),(v, u),(v, s),(z, 9),(z, u),(z, v),(z, s),(s, 9),(s, v)$. By the similar arguments as before one gets $(x, y)=(7,2),(9,8)$ or $(z, u)$.

Subcase 7.7.4.1. $\operatorname{lk}(4)=C_{6}(w, 5,0,3,9,8)$. Completing successively, we get $\operatorname{lk}(8)$ $=C_{6}(6,1,9,4, w, v), \operatorname{lk}(9)=C_{6}(7,1,8,4,3, s), \operatorname{lk}(3)=C_{6}(2,0,4,9, s, z), \operatorname{lk}(2)=$ $C_{6}(7,1,0,3, z, u), \operatorname{lk}(u)=C_{6}(5,6, v, 7,2, z), \operatorname{lk}(7)=C_{6}(9,1,2, u, v, s), \operatorname{lk}(v)=$ $C_{6}(8,6, u, 7, s, w)$ and $\operatorname{lk}(w)=C_{6}(5,4,8, v, s, z)$. Here $M \cong T_{15,1,3}$ by the map $\varphi \circ(0,6,9,4,7,1,5)(u, w)$. 
Subcase 7.7.4.2. $\operatorname{lk}(4)=C_{6}(w, 5,0,3, z, u)$. Completing successively, we get $\operatorname{lk}(u)$ $=C_{6}(6,5, z, 4, w, v), \operatorname{lk}(z)=C_{6}(w, 5, u, 4,3, s), \operatorname{lk}(w)=C_{6}(u, 4,5, z, s, v), \operatorname{lk}(3)=$ $C_{6}(2,0,4, z, s, 9), \operatorname{lk}(9)=C_{6}(3,2,8,1,7, s), \operatorname{lk}(s)=C_{6}(w, z, 3,9,7, v), \operatorname{lk}(v)=$ $C_{6}(6, u, w, s, 7,8)$ and $\operatorname{lk}(7)=C_{6}(2,1,9, s, v, 8)$. Here $M \cong Q_{5,3}$ by the map $\psi \circ$ $(0, z, w, 9,3, s, 6,8,5, u)(4, v, 7)$, where $\psi$ is as in Subcase 2.1 .

Subcase 7.7.4.3. $1 \mathrm{k}(4)=C_{6}(w, 5,0,3,7,2)$. Then, completing successively, we get $\operatorname{lk}(2)=C_{6}(3,0,1,7,4, w), \operatorname{lk}(3)=C_{6}(7,4,0,2, w, s), \operatorname{lk}(7)=C_{6}(1,2,4,3, s, 9)$, $\operatorname{lk}(w)=C_{6}(5,4,2,3, s, z), \operatorname{lk}(s)=C_{6}(9,7,3, w, z, v), \operatorname{lk}(v)=C_{6}(u, 6,8, z, s, 9)$, $\operatorname{lk}(z)=C_{6}(5, w, s, v, 8, u)$ and $\operatorname{lk}(8)=C_{6}(9,1,6, v, z, u)$. Here $M \cong B_{3,5}$ by the map $\psi_{35} \circ(0,9,1,5)(2,8)(3, v)(4,7)(u, w)$, where $\psi_{35}$ is as in Case 1.

Proof of Theorem 6. Let $M$ be an $n$-vertex degree-regular combinatorial 2-manifold of Euler characteristic 0 . Let $d$ be the degree of each vertex. Then $n d=2 f_{1}(M)=3 f_{2}(M)$ and $n-f_{1}(M)+f_{2}(M)=0$. These imply that $d=6$. Now, if $n \in\{12,14,15\}$ then, by Lemmas 3.10-3.12, $M$ is isomorphic to $T_{12,1,2}, \ldots, T_{12,1,4}, T_{6,2,2}, T_{14,1,2}, T_{14,1,3}, Q_{7,2}$, $T_{15,1,2}, \ldots, T_{15,1,5}, Q_{5,3}, B_{3,4}, B_{4,3}, B_{3,5}, B_{5,3}$ or $K_{3,4}$.

Since $B_{3,4}, B_{4,3}, B_{3,5}, B_{5,3}, Q_{7,2}, Q_{5,3}$ and $K_{3,4}$ are non-orientable and remaining 10 are orientable, the second and third statements follow from Lemma 2.1(b), (d), (f), Lemma 2.3(b) and Lemma 2.5(a), (b).

The last statement follows from the fact that $B_{m, n}, K_{m, 2 k}, Q_{2 k+1, n}$ are not weakly regular and $Q_{2 k+1,2}$ is weakly regular for all $m, n \geq 3$ and $k \geq 2$.

\section{Acknowledgements}

The authors are thankful to B Bagchi, S P Inamdar and N S N Sastry for useful conversations. The authors thank the anonymous referee for many useful comments which led to substantial improvements in the presentation of this paper. Theorem 1 was conjectured by the referee. The second author thanks CSIR, New Delhi, India for its research fellowship (Award No.: 9/79(797)/2001-EMR-I).

\section{References}

[1] Altshuler A, Polyhedral realization in $\mathbb{R}^{3}$ of triangulations of the torus and 2-manifolds in cyclic 4-polytopes, Discrete Math. 1 (1971) 211-238

[2] Altshuler A, Construction and representation of neighbourly manifolds, J. Comb. Th. (A) 77 (1997) 246-267

[3] Altshuler A, Bokowski J and Schuchert P, Neighbourly 2-manifolds with 12 vertices, $J$. Comb. Th. (A) 75 (1996) 148-162

[4] Bagchi B and Datta B, A structure theorem for pseudomanifolds, Discrete Math. 168 (1998) 41-60

[5] Datta B and Nilakantan N, Equivelar polyhedra with few vertices, Discrete Comput Geom. 26 (2001) 429-461

[6] Datta B and Upadhyay A K, Degree-regular triangulations of the double-torus Forum. Math. (to appear)

[7] Jungerman M and Ringel G, Minimal triangulations on orientable surfaces, Acta Math. 145 (1980) 121-154 
[8] Lutz F H, Triangulated manifolds with few vertices and vertex-transitive group actions, Thesis (TU, Berlin) (Aachen: Shaker Verlag) (1999)

[9] Ringel G, Wie man die geschlossenen nichtorientierbaren Flächen in möglichst wenig Dreiecke zerlegen kann, Math. Ann. 130 (1955) 317-326

[10] Schulte E and Wills J M, A polyhedral realization of Felix Klein's map $\{3,7\}_{8}$ on a Riemann surface of genus 3, J. London Math. Soc. 32 (1985) 539-547

[11] Schulte E and Wills J M, Geometric realizations for Dyck's regular map on a surface of genus 3, Discrete Comput. Geom. 1 (1986) 141-153

[12] Spanier E H, Algebraic Topology (New York: Springer-Verlag) (1966) 